Classification

Physics Abstracts

13.36

\title{
ANALYSE DES EXPÉRIENCES DE DIFFUSION D'ÉLECTRONS MÉTHODE ET ÉTUDE CRITIQUE (*)
}

\author{
J. BERTHOT et D. B. ISABELLE \\ Laboratoire de Physique Corpusculaire, Université de Clermont \\ 34, avenue Carnot, F63-Clermont-Ferrand \\ (Reçu le 10 mai 1972, révisé le 16 juin 1972)
}

\begin{abstract}
Résumé. - Après avoir présenté une méthode d'analyse des spectres de diffusion des électrons ne faisant intervenir aucun modèle nucléaire, nous discutons les moyens permettant de vérifier la valeur des résultats obtenus. Dans une seconde partie, nous décrivons les diverses mesures effectuées pour établir les domaines de validité des formules utilisées.
\end{abstract}

\begin{abstract}
We describe a method used to analyse spectra of scattered electrons without inclusion of a nuclear model and we discuss the various ways providing a check on the validity of the results. In the second part, we present the results of our measurements to establish the validity range of available formulas.
\end{abstract}

Introduction. - La diffusion élastique ou inélastique des électrons s'est révélée être une méthode d'étude des propriétés des noyaux particulièrement intéressante [1]. En effet, les électrons interagissant d'une manière purement électromagnétique, les techniques de l'électrodynamique quantique permettent de calculer les interactions du champ de l'électron avec les champs électriques et magnétiques associés au noyau. Toutefois, la diffusion des électrons présente l'inconvénient d'être toujours accompagnée par des phénomènes radiatifs qui viennent perturber les mesures expérimentales. En effet, l'électron peut émettre un (ou plusieurs) photons soit dans le champ du noyau diffuseur, ce que nous appellerons par la suite "rayonnement de freinage interne», soit dans le champ d'un noyau du matériau cible ou « rayonnement de freinage externe $»$. Il faut aussi tenir compte $\mathrm{du}$ phénomène d'ionisation des atomes de la cible par l'électron incident ou diffusé.

Les corrections afférentes à ces phénomènes peuvent être classées en deux groupes : d'une part, ce qu'il est convenu d'appeler «les corrections radiatives » et d'autre part, les «queues radiatives». La premiè e correction permet de tenir compte des électrons qui, ayant diffusé dans les bonnes conditions cinématiques (énergie incidente, angle de diffusion, énergie transférée au noyau), n'ont pu être détectés par le dispositif expérimental de résolution finie, par suite de la perte d'une partie de leur énergie après la diffusion.

(*) Ce travail représente une partie de la thèse présentée par J. Berthot en vue de l'obtention du grade de Docteur ès-Sciences Physique.
Les extensions des pics de diffusion vers les basses énergies finales sont communément appelées «queues radiatives ». Ces contributions parasites doivent être soustraites du spectre expérimental avant que celui-ci puisse être comparé aux prédictions d'un modèle nucléaire.

Nous disposons actuellement de formules théoriques pour calculer ces divers phénomènes. Toutefois, un double problème se pose : d'une part, il faut élaborer une méthode précise de correction, d'autre part, il faut étudier quels sont les domaines de validité des formules employées. En effet, les dispositifs expérimentaux actuellement utilisés pour la diffusion des électrons permettent des mesures d'une telle qualité qu'il est indispensable de savoir avec quelle précision il est possible de traiter ces corrections.

Cet article a pour but d'apporter une solution aussi complète que possible à ces deux aspects du problème. Après avoir fait un rappel des formules et des approximations couramment utilisées, nous présentons notre programme d'analyse des données expérimentales. Le processus suivi est similaire à celui déjà proposé par divers auteurs [2], [3] mais présente toutefois par rapport aux précédents, l'avantage de ne faire intervenir aucun modèle nucléaire. Dans la troisième partie, sont discutées les différentes méthodes permettant de vérifier la précision de notre analyse dans le cas d'un noyau léger $\left({ }^{12} \mathrm{C}\right)$. Enfin, dans la dernière partie, sont discutées les formules de correction tenant compte des termes d'ordre supérieur à ceux de la première approximation de Born. Les domaines de validité de ces corrections seront établis à partir des résultats expérimentaux obtenus pour trois noyaux : le carbone 12, l'étain 83 et le bismuth 209. 
La méthode employée pour l'analyse est une méthode de "déconvolution». Remarquons ici qu'un procédé plus précis consiste à faire une convolution de la section efficace théorique par les divers phénomènes introduisant une distorsion [4]. Le spectre calculé ainsi obtenu peut être directement comparé au spectre expérimental. Toutefois, une telle méthode implique la connaissance exacte des fonctions d'ondes nucléaires et n'est donc applicable que pour les noyaux simples tels que protons, deutérium, tritium ou hélium. On peut espérer cependant que dans un avenir proche, les progrès réalisés dans la connaissance des modèles nucléaires permettront l'emploi d'une telle méthode pour des noyaux plus complexes comme le carbone ou l'oxygène.

I. Généralités et notations. - Dans un spectre obtenu avec des électrons incidents d'énergie $E_{\mathrm{i}}, \mathbf{p}_{\mathrm{i}}$, considérons un point expérimental correspondant à des électrons diffusés sous un angle $\theta$ avec une énergie finale $E_{\mathrm{f}}, \mathbf{p}_{\mathrm{f}}$.

L'énergie $\omega$ transférée au noyau ou énergie d'excitation est donnée par :

$$
\omega=E_{\mathrm{i}}-E_{\mathrm{f}}-\frac{q^{2}}{2 M_{\mathrm{T}}}
$$

où $\mathbf{q}=\mathbf{p}_{\mathrm{i}}-\mathbf{p}_{\mathrm{f}}$ est la $\cdot$ quantité de mouvement transférée au noyau et $M_{\mathrm{T}}$ la masse du noyau cible.

Désignons par $\sigma_{\mathrm{ex}}\left(E_{\mathrm{i}}, \omega, \theta\right)$ la section efficace doublement différentielle mesurée et par $\sigma\left(E_{\mathrm{i}}, \omega, \theta\right)$ la même section efficace corrigée des effets radiatifs, c'est-à-dire celle pouvant être comparée aux prédictions des modèles nucléaires.

La relation entre $\sigma_{\text {ex }}$ et $\sigma$ est obtenue en tenant compte du fait que l'électron peut perdre une quantiét $k$ d'énergie soit avant (Fig. 1a), soit après (Fig. 1b) la diffusion nucléaire. Nous avons alors quel que soit le processus produisant cette perte d'énergie :

$$
\begin{aligned}
& \sigma\left(E_{\mathrm{i}}, \omega, \theta\right)=\left\{\sigma_{\mathrm{ex}}\left(E_{\mathrm{i}}, \omega, \theta\right)-\right. \\
& \quad-\int_{E_{\mathrm{f}}}^{E_{\mathrm{i}}-\Delta} \varphi\left(E_{\mathrm{i}}, E_{\mathrm{i}}^{\prime}\right) \sigma\left(E_{\mathrm{i}}^{\prime}, \omega, \theta\right) \mathrm{d} E_{\mathrm{i}}^{\prime} \\
& \left.-\int_{E_{\mathrm{f}}+\Delta}^{E_{\mathrm{i}}} \varphi\left(E_{\mathrm{f}}^{\prime}, E_{\mathrm{f}}\right) \sigma\left(E_{\mathrm{i}}, \omega^{\prime}, \theta\right) \mathrm{d} E_{\mathrm{f}}^{\prime}\right\} \varepsilon\left(E_{\mathrm{i}}, \omega, \theta\right)
\end{aligned}
$$

avec :

$$
\omega^{\prime}=E_{\mathrm{i}}-E_{\mathrm{f}}^{\prime} .
$$

La fonction $\varphi\left(E, E^{\prime}\right)$ représente la probabilité pour qu'un électron d'énergie $E$ perde une énergie $k=E-E^{\prime}$ soit par rayonnement de freinage, soit par ionisation. Les deux intégrales de l'éq. (2) correspondent aux contributions des «queues radiatives" respectivement avant et après la diffusion nucléaire. L'énergie $\Delta$ qui apparaît aux bornes des intégrales ci-dessus est la limite arbitraire de séparation des photons réels

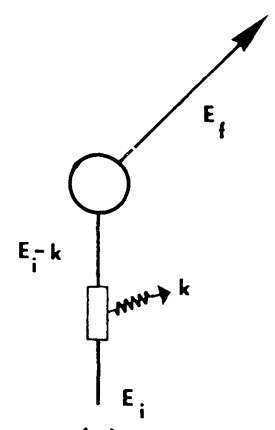

(a)

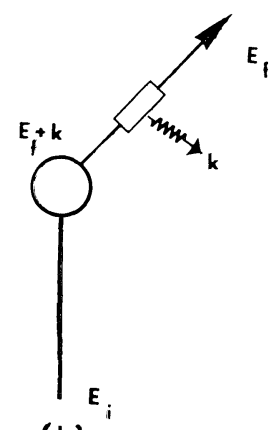

(b)
FIG. 1.

«mous» correspondant aux corrections radiatives et des photons réels "durs » correspondant aux queues radiatives.

La correction radiative proprement dite, telle qu'elle a été définie plus haut, peut être mise sous la forme $\mathrm{du}$ facteur multiplicatif $\varepsilon^{\prime}\left(E_{\mathrm{i}}, \vdots \omega, \theta\right)$ (voir éq. (3), (5) et (8)).

II. Rappels théoriques. - II.1. FoRMULATION DES CORRECTIONS RADIATIVES. - Cette correction correspond aux électrons qui, ayant diffusé à la bonne énergie $E_{\mathrm{f}}$, ne sont pas détectés par le dispositif expérimental soit par suite d'une réaction de l'électron sur son champ propre, soit du fait d'une perte d'énergie de l'électron diffusé, par rayonnement de freinage ou par ionisation.

II.1.1. Correction dite de "Schwinger ». '- Elle provient du fait que nous ne pouvons mesurer directement le diagramme (a) de la figure 2, mais seulement la somme des diagrammes $(a)$ et $(b)$. Les trois premiers diagrammes $(b)$ tiennent compte de l'effet réactif de l'électron sur son champ propre et le quatrième de la polarisation du vide. Les deux derniers diagrammes correspondant à l'émission de photons mous d'énergie plus petite que la résolution expérimentale $\Delta E$, doivent être inclus afin d'éliminer la divergence infrarouge. Ces termes ont été tout d'abord calculés par Schwinger [6] puis repris par Yennie et al. [7].

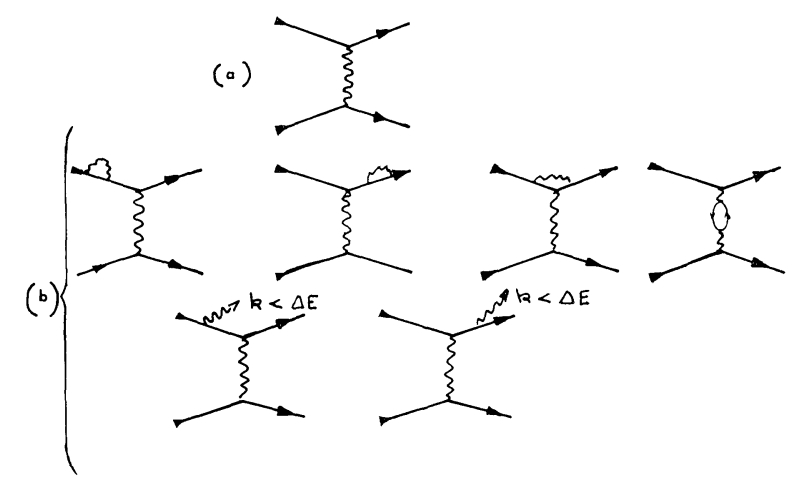

FIG. 2. - Diagrammes correspondant aux corrections radiatives. 
La section efficace de diffusion élastique par un champ coulombien mesurée expérimentalement est reliée à la section efficace corrigée de ces effets par :

$$
\sigma\left(E_{\mathrm{i}}, \omega, \theta\right)=\sigma_{\mathrm{ex}}\left(E_{\mathrm{i}}, \omega, \theta\right) \cdot \mathrm{e}^{\delta_{\mathrm{s}}}
$$

où

$$
\begin{aligned}
\delta_{\mathrm{s}}=\frac{4 \alpha}{\pi} \cdot\left(\left(l_{\mathrm{n}} \frac{2 E_{\mathrm{i}} \sin \theta / 2}{m c^{2}}-\frac{1}{2}\right) \times\right. \\
\left.\quad \times\left(l_{\mathrm{n}} \frac{E_{\mathrm{i}}}{\Delta E}-\frac{13}{12}\right)+\frac{17}{12}+L(\theta)\right)
\end{aligned}
$$

et où $\Delta E$ est la résolution expérimentale. L'éq. (3) ne correspond pas uniquement aux seuls diagrammes de la figure 2, mais inclus également ceux d'ordre supérieur correspondant notamment à l'émission de plusieurs photons.

La fonction $L(\theta)$ est donnée sous la forme d'une intégrale et ne dépend que de l'angle de diffusion $\theta$. Elle a été tabulée par Lacoste [8].

Dans le cas de la diffusion inélastique, la correction radiative a été calculée par Meister et Griffy [9] et par Perez Y Jorba [22] et discutée par Maximon [10]. L'expression obtenue ne diffère de la précédente que par un terme additionnel, $\frac{1}{4} l_{\mathrm{n}}\left(E_{\mathrm{i}} / E_{\mathrm{f}}\right)^{2}$ terme qui est toujours petit.

II.1.2. Corrections dues à l'épaisseur finie des cibles. - A la correction radiative proprement dite il y a lieu d'ajouter les effets produisant un élargissement du spectre (straggling) dû au rayonnement de freinage et aux collisions avec les électrons atomiques.

Nous tiendrons compte du premier effet, en écrivant qu'entre les sections efficaces mesurées et corrigées, nous avons la relation:

$$
\sigma\left(E_{\mathrm{i}}, \omega, \theta\right)=\sigma_{\mathrm{ex}}\left(E_{\mathrm{i}}, \omega, \theta\right) \cdot \mathrm{e}^{\delta_{\mathrm{B}}}
$$

avec :

$$
\mathrm{e}^{-\delta_{\mathbf{B}}}=\int_{0}^{\Delta E} \varphi\left(E, E^{\prime}\right) \mathrm{d} k
$$

où $\varphi\left(E, E^{\prime}\right) \mathrm{d} k$ est la probabilité pour qu'un électron d'énergie $E$ émette un photon d'énergie comprise entre $k=E-E^{\prime}$ et $k+\mathrm{d} k$.

En utilisant les formules données par Rossi [11], pour $\Delta E \ll E$, le terme correctif $\delta_{\mathrm{B}}$ s'écrit :

$$
\delta_{\mathrm{B}} \simeq \frac{t}{\chi_{0} l_{\mathrm{n}} 2} l_{\mathrm{n}} \frac{E}{\Delta E}
$$

où $\chi_{0}$ est la longueur de radiation du matériau considéré et $t$ l'épaisseur de la cible.

Le calcul des effets de straggling dus à l'ionisation a été réalisé dans le cas général par Landau [12]. Nous avons suivi la procédure et les formules analytiques proposées par Nguyen Ngnoc [13] qui a montré qu'entre les sections efficaces mesurées et corrigées, nous avons la relation :

$$
\sigma\left(E_{\mathrm{i}}, \omega, \theta\right)=\sigma_{\mathrm{ex}}\left(E_{\mathrm{i}}, \omega, \theta\right)\left(1+\delta_{\mathrm{I}}\right)
$$

où $\delta_{\mathrm{I}}$ ne dépend que de $E_{\mathrm{i}}, t$ et $\Delta E$. Son expression analytique est développée dans les références [13], [24].

II.2. Les Queues Radiatives. - L'extension des pics de diffusion vers les basses énergies finales est due aussi bien au rayonnement de freinage interne, qu'au rayonnement de freinage externe et à l'ionisation.

II.2.1. Rayonnement de freinage interne. - Le calcul des contributions correspondant aux diagrammes $(a)$ et $(b)$ de la figure 3 , est effectué en utilisant l'expression proposée par Maximon et Isabelle [14].
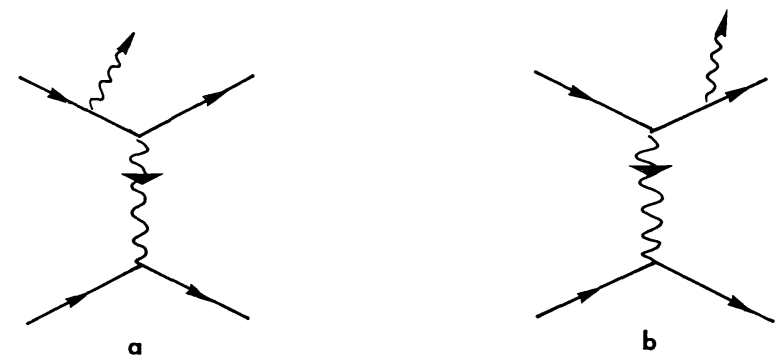

FIG. 3. - Diagrammes correspondant au rayonnement de freinage interne.

Elle est obtenue en intégrant sans approximation la section efficace de Bethe-Heitler [15] sur tous les angles d'émission du photon. Le résultat est exprimé en fonction des facteurs de forme nucléaires laissés sous forme implicite et peut se mettre sous la forme :

$$
\frac{\mathrm{d}^{2} \sigma}{\mathrm{d} \Omega \mathrm{d} k}=\frac{1}{2 \pi} \cdot \frac{e^{2}}{\hbar c}\left(\frac{Z e^{2}}{m c^{2}}\right)^{2} \frac{1}{k} \cdot \frac{1}{E_{\mathrm{i}}^{2}}(P+B)
$$

où $k$ est l'énergie du photon émis.

Le terme $P$, dit terme « de pic », est la contribution à la section efficace, des photons émis dans la direction soit de l'électron incident, soit de l'électron diffusé. Le terme $B$ est la contribution des photons émis dans toutes les autres directions.

D'après Maximon-Isabelle [14], $P$ est mis sous la forme :

$$
\begin{aligned}
P= & \frac{1}{2} \frac{\cos ^{2} \theta / 2}{\sin ^{4} \frac{\theta}{2}} \cdot\left[S ( q _ { 2 } ) \left(\frac{E_{\mathrm{i}}^{2}+\left(E_{\mathrm{f}}+\omega\right)^{2}}{\left(E_{\mathrm{f}}+\omega\right)^{2}} \times\right.\right. \\
& \left.\times l_{\mathrm{n}}\left(\frac{2 E_{\mathrm{i}}}{m c^{2}}\right)-\frac{E_{\mathrm{i}}}{E_{\mathrm{f}}+\omega}\right)+S\left(q_{1}\right)\left(\frac{E_{\mathrm{f}}^{2}+\left(E_{\mathrm{i}}-\omega\right)^{2}}{\left(E_{\mathrm{i}}-\omega\right)^{2}}\right. \\
& \left.\left.\times l_{\mathrm{n}}\left(\frac{2 E_{\mathrm{f}}}{m c^{2}}\right)-\frac{E_{\mathrm{f}}}{E_{\mathrm{i}}-\omega}\right)\right]
\end{aligned}
$$

où $q_{1}$ et $q_{2}$ sont les transferts de quantité de mouvement correspondant respectivement aux diagrammes $(a)$ et $(b)$ de la figure 3. 
Si $E \gg m c^{2}$, on peut écrire :

$$
\begin{aligned}
& q_{1}^{2} \sim \omega^{2}+4 E_{\mathrm{i}}\left(E_{\mathrm{i}}-\omega\right) \sin ^{2} \frac{\theta}{2} \\
& q_{2}^{2} \sim \omega^{2}+4 E_{\mathrm{f}}\left(E_{\mathrm{f}}+\omega\right) \sin ^{2} \frac{\theta}{2} .
\end{aligned}
$$

$\omega$ est l'énergie d'excitation correspondant aux diagrammes $(a)$ et $(b)$ de la figure 3 tandis que $S\left(q_{1}\right)$ et $S\left(q_{2}\right)$ sont des facteurs de forme nucléaires correspondant aux transferts $q_{1}$ et $q_{2}$. Ils sont reliés aux facteurs de forme longitudinal $F_{\mathrm{L}}$ et transverse $F_{\mathrm{T}}$ par la relation :

$$
\begin{aligned}
S(q)=(1-\beta)^{2} F_{\mathrm{L}}^{2}(q)+(1-\rho) & F_{\mathrm{T}}^{2}(q)+ \\
& +2 F_{\mathrm{T}}^{2}(q) \operatorname{tg}^{2} \frac{\theta}{2}
\end{aligned}
$$

avec $\beta=\omega^{2} / q^{2}$.

Le terme $B$ explicité dans la référence [14] est mis sous la forme d'une somme d'intégrales de fonctions des facteurs de forme $F_{\mathrm{L}}$ et $F_{\mathrm{T}}$.

II.2.2. Rayonnement de freinage externe. - La probabilité différentielle pour qu'un électron d'énergie $E$ émette un photon d'énergie comprise entre $k$ et $k+\mathrm{d} k$, en traversant une épaisseur $\mathrm{d} t$ de matière est donnée d'après Bethe et Heitler [15] par :

$$
\varphi_{r}(E, k) \mathrm{d} k . \mathrm{d} t=4 \frac{Z^{2}}{A} N \alpha r_{0}^{2} \frac{1}{k} F(E, v) \mathrm{d} k \cdot \mathrm{d} t
$$

où :

$$
\begin{aligned}
v & =k / E \\
\alpha & =e^{2} / \hbar c \\
A & =\text { nombre de masses } \\
Z & =\text { numéro atomique } \\
r_{0} & =\text { rayon classique de l'électron } \\
N & =\text { nombre d'Avogadro. }
\end{aligned}
$$

L'effet d'écran des électrons atomiques est introduit par la fonction $F(E, v)$. L'expression analytique de cette fonction a été explicitée par de nombreux auteurs [11], [15], [16], [23].

II.2.3. Collisions dues aux électrons atomiques. La probabilité pour qu'un électron d'énergie $E$ perde par collision avec les électrons atomiques une quantité d'énergie comprise entre $k$ et $\mathrm{d} k+k$ en traversant une épaisseur $\mathrm{d} t$ de matière est donnée par Moller [17] :

$$
\begin{aligned}
\varphi_{\text {coll }}(E, k) \mathrm{d} k \cdot \mathrm{d} t=2 \pi N \frac{Z}{A} & r_{0}^{2} m c^{2} \mathrm{~d} k \cdot \mathrm{d} t \times \\
& \times\left\{\frac{(1-v)^{2}+v}{v(1-v)}\right\}^{2}
\end{aligned}
$$

II.3. Approximations. - II.3.1. Approximation dite «de pic» (ou «peaking approximation»). Dans l'expression (10), le terme $P$ dû à l'émission de photons le long de la trajectoire de l'électron est séparé du terme $B$ qui correspond à l'émission de photons dans toutes les autres directions. L'approximation du " pic », introduite par Schiff [18] et utilisée par de nombreux auteurs, revient à négliger le terme $B$ devant le terme $P$. Or, ce terme $B$ devient importante $(B>0,1 P)$ dès que la perte d'énergie $k$ devient grande par rapport à l'énergie de l'électron $(k>E / 3)$ [5].

Dans notre analyse, nous tiendrons compte du terme $B$, uniquement pour le calcul de l'extension des pics élastiques vers les basses énergies finales. Nous le négligerons dans l'évaluation de l'extension des pics inélastiques et quasi élastiques. Ceci est justifié car dans ce cas, la queue radiative n'est importante qu'au voisinage immédiat des pics inélastiques. En effet, à la décroissance en $1 / k$ de la section efficace radiative, s'ajoute la décroissance des facteurs de forme inélastiques, facteurs de forme qui tendent vers zéro quand $q$ devient petit. D'autre part, nous avons limité nos mesures expérimentales à des angles de diffusion inrerieurs à $120^{\circ}$, le terme $B$ devenant très grand lorsque la diffusion s'effectue vers l'arrière.

II.3.2. Approximation de Born. - Toutes les formules ci-dessus ont été établies en première approximation de Born. Une forme simple de correction d'ordre $\alpha Z$ à la formule de Bethe-Heitler a été proposée par E. Borie [19] dans le cas élastique.

Toutefois, cette expression est établie en utilisant l'approximation de " pic » et n'est donc valable que pour la correction du terme $P$ de l'éq. (10). Nous. discuterons ce point plus en détail dans la partie V.

III. pescription du programme de déconvolution. Afin de disposer de valeurs expérimentales pour les sections efficaces, nous avons mesuré pour un angle de diffusion donné, un ensemble de spectres expérimentaux correspondant à plusieurs valeurs de l'énergie incidente. Nous obtenons ainsi par points. une hypersurface donnant la valeur de la section efficace pour toutes les valeurs mesurées du couple $\left(E_{\mathrm{i}}, E_{\mathrm{f}}\right)$ à un angle donné. La base de cette surface dans le plan $\left(E_{\mathrm{i}}, E_{\mathrm{f}}\right)$ constitue le triangle canonique (Fig. 4). Lors du calcul des queues radiatives (éq. 2), nous aurons besoin de la valeur de $\sigma$ pour des valeurs de $\left(E_{\mathrm{i}}, E_{\mathrm{f}}\right)$ différentes de celles mesurées, nous serons. donc amenés à faire des interpolations (ou extrapolations) entre les points expérimentaux.

Or, pour une énergie incidente donnée, la section efficace présente en fonction de $\omega$ des extrema. Par contre, pour une valeur donnée de $\omega$ la section efficace varie d'une façon monotone en fonction de l'énergie incidente. En conséquence, les interpolations (ou extrapolations) seront toujours faites le long de lignes telles que dans le plan $\left(E_{\mathrm{i}}, E_{\mathrm{f}}\right) \omega$ soit constant (lignes iso- $\omega$ sur la Fig. 4).

Chaque spectre est divisé en bandes d'énergie de largeur $\Delta$ bandes que nous assimilons à des pics. Nous avons toujours pris pour cette largeur une valeur voisine de la résolution expérimentale. Toutefois, un calcul numérique nous a montré que le résultat final est pratiquement indépendant de ce choix. Ce résultat 


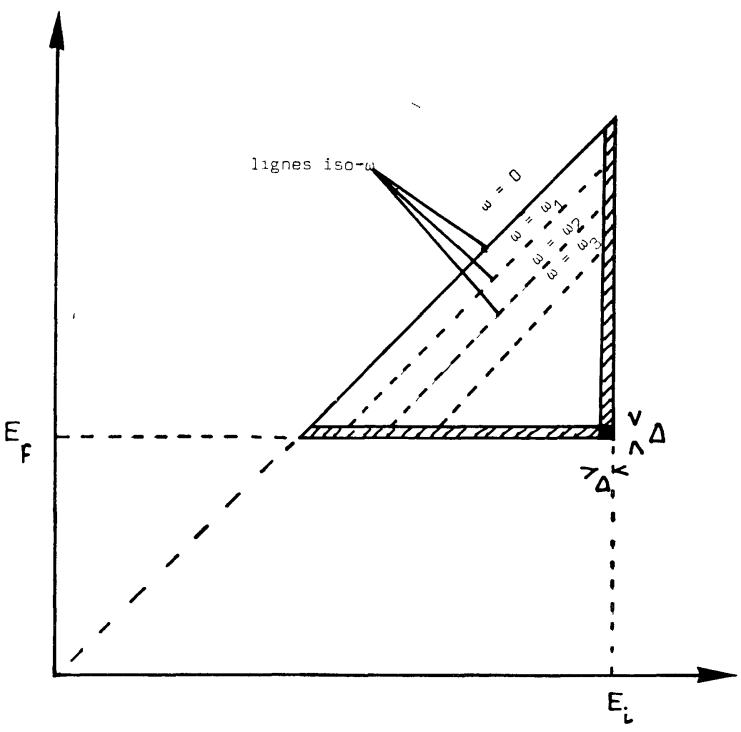

FIG. 4. - Triangle canonique.

est en accord avec le fait que la dérivée de l'éq. (2) par rapport à $\Delta$ est nulle, tant que les sections efficaces peuvent être considérées comme constantes dans l'intervalle d'énergie $\Delta$.

Désignons par $\omega_{j}$ l'énergie d'excitation moyenne de la bande d'énergie à laquelle appartient le point expérimental à corriger et par $\omega_{m}$ l'énergie d'excitation moyenne de la bande d'énergie correspondant à une diffusion nucléaire suivie ou précédée d'une perte d'énergie $k$ telle que :

$$
k=\omega_{j}-\omega_{m} .
$$

$\mathrm{Ne}$ considérant que des ensembles de spectres pris à un angle $\theta$ donné, nous ne mentionnerons plus cette variable dans les définitions des grandeurs considérées. D'autre part, utilisant pour l'intégration un pas fini, nous écrirons l'éq. (2) sous la forme :

$$
\sigma\left(E_{l}, \omega_{j}\right)=\left[\sigma_{\mathrm{ex}}\left(E_{l}, \omega_{j}\right)-\sum_{m=1}^{j-1} T_{m j}\right] \varepsilon\left(E_{l}, \omega_{j}\right)
$$

avec :

$$
\begin{aligned}
T_{m j} & =\varphi\left(E_{l}-\omega_{m}, E_{l}-\omega_{j}\right) \cdot \sigma\left(E_{l}, \omega_{m}\right)+ \\
& +\varphi\left(E_{l}, E_{l}-\left(\omega_{j}-\omega_{m}\right)\right) \cdot \sigma\left(E_{l}-\left(\omega_{j}-\omega_{m}\right), \omega_{m}\right.
\end{aligned}
$$

tandis que $E_{l}$ est l'énergie incidente correspondant au $l$-ième spectre.

L'erreur statistique associée à la détermination de $\sigma\left(E_{l}, \omega_{j}\right)$ est donnée par :

$$
\begin{aligned}
& \Delta \sigma\left(E_{l}, \omega_{j}\right)= \\
& \quad=\left[\left(\Delta \sigma_{\mathrm{ex}}\left(E_{l}, \omega_{j}\right)\right)^{2}+\sum_{m-1}^{j-1}\left(\Delta T_{m j}\right)^{2}\right]^{1 / 2} \varepsilon\left(E_{l}, \omega_{j}\right)
\end{aligned}
$$

où $\Delta T_{m j}$ est calculé au moyen de l'éq. (17) en introduisant les erreurs statistiques afférentes à $\sigma\left(E_{l}, \omega_{m}\right)$ et à $\sigma\left(E_{l}-\left(\omega-\omega_{m}\right), \omega_{m}\right)$.
Les séquences du calcul sont alors les suivantes :

1) Calcul pour les $l$ spectres de la section efficace corrigée pour $j=1\left(\omega_{j}=\omega_{1}\right)$

$$
\sigma\left(E_{l}, \omega_{1}\right)=\sigma_{\mathrm{ex}}\left(E_{l}, \omega_{1}\right) \cdot \varepsilon\left(E_{l}, \omega_{1}\right)
$$

et de l'erreur correspondante $\Delta \sigma\left(E_{l}, \omega_{1}\right)$.

2) Calcul pour chacun des spectres de l'extension vers les basses énergies de la bande centrée en $\omega_{1}$ (queue élastique), puis soustraction de cette contribution pour tous les points ayant une énergie d'excitation $\omega$ supérieure à $\omega_{1}$. Ceci revient à remplacer pour $j>2, \sigma_{\mathrm{ex}}\left(E_{l}, \omega_{j}\right)$ par :

$$
\left\{\sigma_{\mathrm{ex}}\left(E_{l}, \omega_{j}\right)-T_{j 1}\left(E_{l}\right)\right\}
$$

avec :

$T_{j 1}\left(E_{l}\right)=\varphi\left(E_{l}-\omega_{1}, E_{l}-\omega_{j}\right) \cdot \sigma\left(E_{l}, \omega_{1}\right)+$ $+\varphi\left(E_{l}, E_{l}-\left(\omega_{j}-\omega_{1}\right)\right) \cdot \sigma\left(E_{l}-\left(\omega_{j}-\omega_{1}\right), \omega_{1}\right)$.

La section efficace $\sigma\left(E_{l}, \omega_{1}\right)$ est obtenue directement à partir du résultat de la séquence 1 , tandis que $\sigma\left[E_{l}-\left(\omega_{j}-\omega_{1}\right), \omega_{1}\right]$ doit être interpolé ainsi que nous l'avons décrit précédemment.

3) Cette séquence est identique à la séquence 1 , mais pour $j=2$. Elle nous permet de calculer $\sigma\left(E_{l}, \omega_{2}\right)$ donnée par:

$$
\sigma\left(E_{l}, \omega_{2}\right)=\left(\sigma_{\text {ex }}\left(E_{l}, \omega_{2}\right)-T_{21}\right) . \varepsilon\left(E_{l}, \omega_{2}\right)
$$

ainsi que l'erreur correspondante.

4) Cette séquence est identique à la séquence 2 . Elle nous permet de remplacer pour $j>3$ :

$$
\left(\sigma_{\mathrm{ex}}\left(E_{l}, \omega_{j}\right)-T_{j 1}\left(E_{l}\right)\right)
$$

par

$$
\left(\sigma_{\mathrm{ex}}\left(E_{l}, \omega_{j}\right)-T_{j 1}\left(E_{l}\right)-T_{j 2}\left(E_{l}\right)\right)
$$

et ainsi de suite, de proche en proche nous corrigeons tous les spectres correspondant à un angle de diffusion donné. Toutefois, si nous voulons dans notre procédure effectuer le calcul en ne faisant pas l'approximation du pic définie plus haut, il faut pour chaque valeur de $\omega_{j}$ et $\omega_{i i}$ calculer le terme $B$ de l'éq. (9).

Pour ce faire, nous devons disposer de deux ensembles canoniques correspondant à deux angles distincts, aussi différents que possible, afin de pouvoir séparer dans le cas de la première approximation de Born, les parties longitudinales et transverses des sections efficaces.

IV. Vérification expérimentale de la validité du processus d'analyse dans le cas du carbone 12. Nous avons vu dans la première partie que de nombreux auteurs ont proposé des expressions théoriques permettant le calcul des phénomènes radiatifs. Toutefois, très peu abordent les vérifications expérimentales des formules utilisées. Il s'agit en effet d'étudier les domaines de validité des approximations théoriques, à savoir essentiellement la première approximation de Born et l'approximation "du pic». De plus, il 
faut s'assurer que la méthode d'analyse utilisée n'introduit ni distorsion $\mathrm{ni}$ biais systématique dans le spectre corrigé.

Malheureusement, il n'est pas possible par des expériences de diffusion simple électron-noyau, de vérifier séparément l'importance de chacun des termes de la correction et la validité de chaque approximation. Nous devons nous contenter d'une vérification globale, vérification que nous avons effectuée par diverses méthodes sur des spectres de diffusion sur le carbone 12.

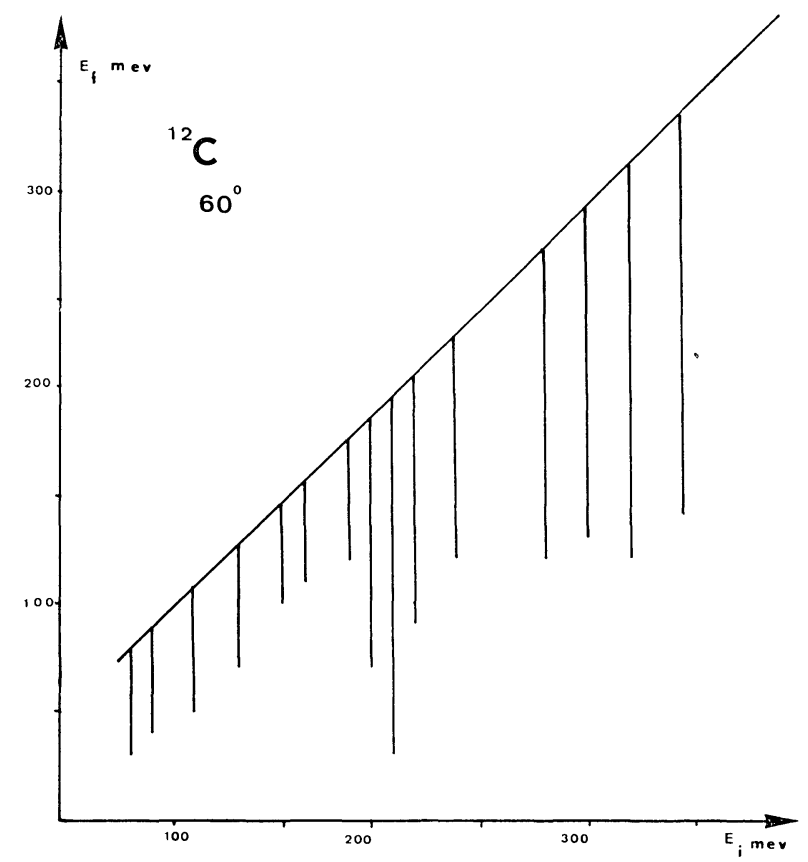

FIG. 5. - Triangle canonique utilisé pour la correction des spectres de diffusion à $60^{\circ}$ sur le carbone. Les lignes verticales représentent dans le plan $\left(E_{i}, E_{f}\right)$ les spectres mesurés à $E_{i}$ constant.

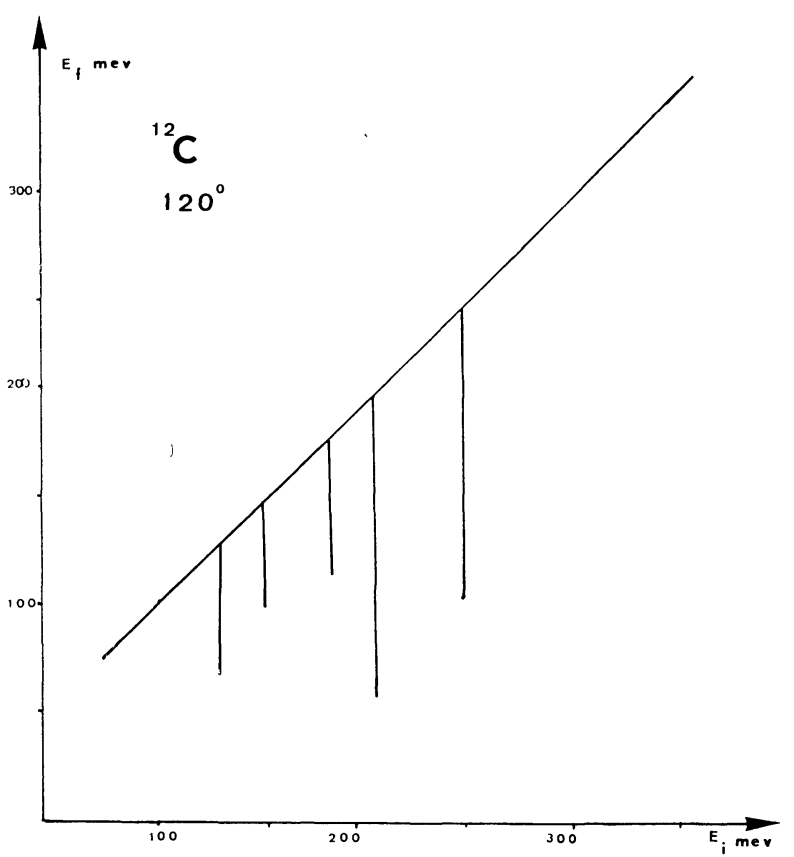

FIG. 6. - Idem pour $\theta=120^{\circ}$ 。
Utilisant un dispositif expérimental classique, constitué par un spectromètre magnétique à double focalisation, avec détecteur monocanal dans le plan focal, nous avons mesuré pour deux angles de diffusion, à savoir 60 et $120^{\circ}$, un certain nombre de spectres de diffusion sur le carbone 12 . L'ensemble des points expérimentaux constitue les deux triangles canoniques des figures 5 et 6 .

Ces expériences ont été effectuées avec une résolution expérimentale de $0,5 \%$ et avec une épaisseur de cible de $0,15 \mathrm{~g} / \mathrm{cm}^{2}$. Chaque point expérimental a été mesuré avec une précision statistique de $6 \%$.

Sur la figure 7, sont comparées à titre d'exemple,

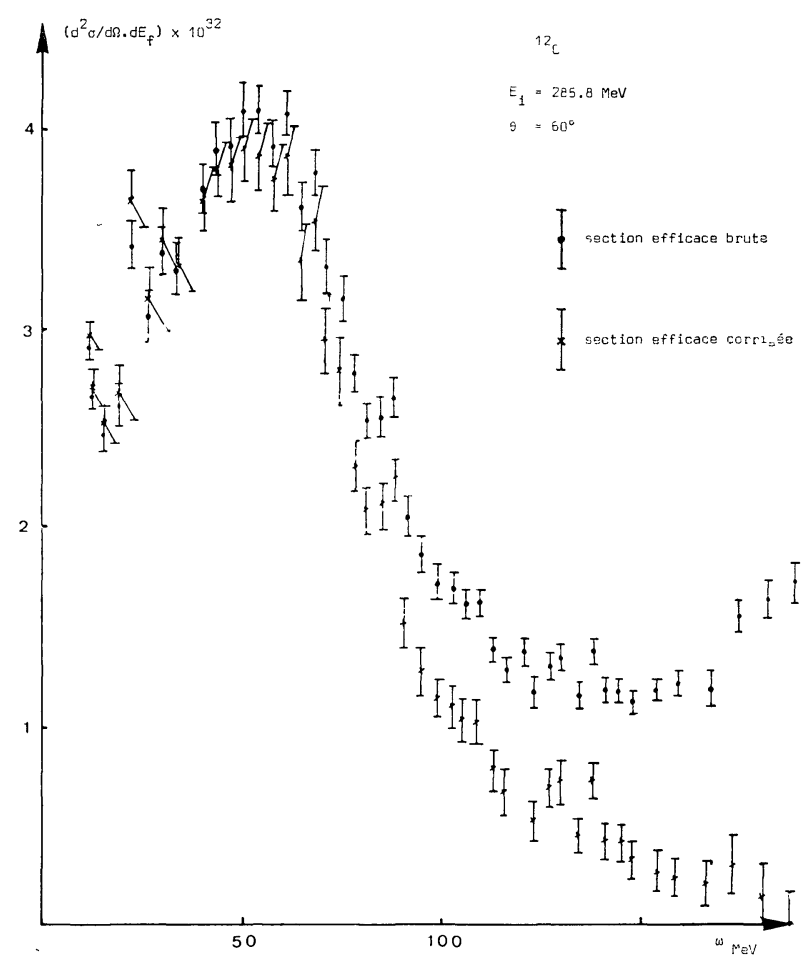

Fig. 7. - Sections efficaces expérimentales et corrigées pour un spectre de diffusion sur le carbone, correspondant à un angle de diffusion de $60^{\circ}$ et une énergie incidente de $285,8 \mathrm{MeV}$.

la section efficace expérimentale et la section efficace corrigée correspondant à un spectre de diffusion à $60^{\circ}$ pour une énergie incidente de $285,8 \mathrm{MeV}$.

La connaissance de la section efficace pour deux angles distincts nous permet d'extraire les facteurs de forme longitudinaux et transverses nécessaires au calcul de terme $B$ de l'expression (9). En effet, l'expression de la section efficace en fonction de $q$ et $\omega$, pouvant s'écrire sous la forme (1) :

$$
\begin{aligned}
\frac{\mathrm{d} \sigma}{\mathrm{d} \Omega}=4 \pi \sigma_{\mathrm{M}}\left\{\left(\frac{q_{\mu}^{2}}{q^{2}}\right)^{2} F_{L}^{2}(q, \omega)+\right. & \\
& \left.+\left(\frac{1}{2}+\frac{q_{\mu}^{2}}{q^{2}} \cdot \operatorname{tg}^{2} \frac{\theta}{2}\right) F_{\mathrm{T}}^{2}(q, \omega)\right\}
\end{aligned}
$$

avec :

$$
q_{\mu}^{2}=q^{2}-\omega^{2}
$$


et

$$
\sigma_{\mathrm{M}}=\left(\frac{Z \alpha}{2 E_{\mathrm{i}}}\right)^{2} \cdot \frac{\cos ^{2} \theta / 2}{\sin ^{4} \theta / 2} \cdot \frac{1}{\left(1+2 E_{\mathrm{i}} \sin ^{2} \theta / 2 / M_{\mathrm{T}}\right)}
$$

nous pouvons obtenir les facteurs de forme $F_{\mathrm{L}}$ et $F_{\mathrm{T}}$ à condition de faire correspondre dans les deux ensembles, les points expérimentaux ayant même $q$ et même $\omega$.

Toutefois, un tel calcul se fait avec une assez mauvaise précision (20 à $30 \%$ ), surtout en ce qui concerne le facteur de forme transverse.

De plus, nous avons mesuré avec une précision statistique de $1 \%$, les spectres en énergie des électrons diffusés à l'angle $\theta=60^{\circ}$, pour une énergie incidente de $213,4 \mathrm{MeV}$ et pour trois épaisseurs de cibles différentes, à savoir :

$$
t_{0_{1}}=0,1569, \quad t_{0_{2}}=0,3217
$$

et

$$
t_{0_{3}}=0,6574 \mathrm{~g} / \mathrm{cm}^{2} .
$$

La vérification expérimentale du calcul de correction est faite par les méthodes suivantes :

IV.1. Test D'INVARIANCE DES SPECTRES PAR RAPPORT AUX PARAMÈTRES EXPÉRIMENTAUX. - Pour s'assurer de la validité de la méthode d'analyse proposée, il faut d'abord vérifier que les spectres expérimentaux corrigés ne dépendent pas des conditions expérimentales (épaisseur de la cible, résolution expérimentale), ni de la largeur du pas d'itération.

La figure 8 montre la distribution en fonction de l'énergie d'excitation de la quantité :

$$
\left[\sigma_{t_{1}}(\omega)-\sigma_{t_{2}}(\omega)\right] \times \frac{100}{\sigma_{t_{1}}}(\omega)
$$

où $\sigma_{t}(\omega)$ est la section efficace corrigée de diffusion sur une cible d'épaisseur $t$.

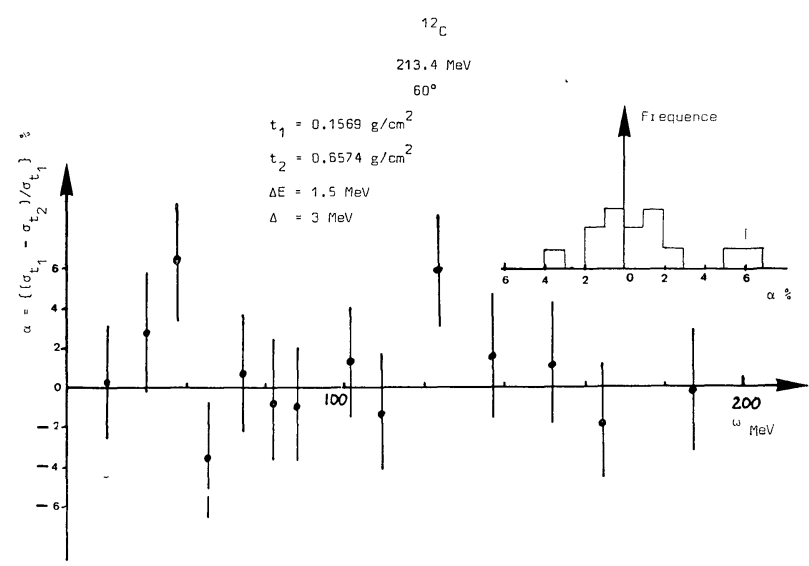

Fig. 8. - Distribution en fonction de $\omega$ de la quantité :

$$
\alpha=\left(\sigma_{t_{1}}-\sigma_{t_{2}}\right) / \sigma_{t_{1}} \text { en } \%=f(\omega) \text {. }
$$

On voit qu'il n'existe aucun biais systématique dans le calcul des termes proportionnels à $t$. En effet, la moyenne de la distribution égale dans ce cas à $(1,1 \pm 2,7) \%$ est comparable à l'erreur statistique obtenue sur la différence de deux sections efficaces mesurées à $1,5 \%$ près, soit $2,3 \%$.

De la même façon nous avons porté sur la figure 9, la distribution en fonction de l'énergie d'excitation de la quantité :

$$
\left(\sigma_{\Delta E_{1}}(\omega)-\sigma_{\Delta E_{2}}(\omega)\right) \times \frac{100}{\sigma_{\Delta E_{1}}}(\omega)
$$

où $\sigma_{\Delta E}(\omega)$ est la section efficace corrigée correspondant à une résolution totale $\Delta E$ de l'appareillage expérimental. $\Delta E_{1}$ et $\Delta E_{2}$ ont été choisis respectivement égaux à 2 et à $10 \mathrm{MeV}$, le pas de l'itération étant égal à $3 \mathrm{MeV}$.

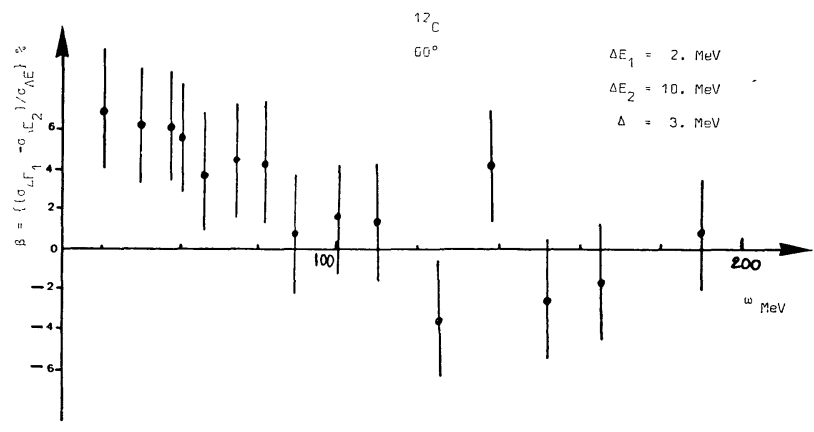

FIG. 9. - Distribution en fonction de $\omega$ de la quantité :

$$
\left(\sigma_{\Delta E_{1}}-\sigma_{\Delta E_{2}}\right) / \sigma_{\Delta E_{1}} \% \text {. }
$$

L'examen de cette distribution montre que sur l'ensemble du spectre, le choix de la résolution expérimentale n'intervient pas dans le résultat final. Il faut remarquer toutefois que dans la région où la «queue radiative » est importante en valeur absolue, il existe une légère divergence entre les résultats. Cette divergence quoique du même ordre de grandeur que l'erreur statistique, provient d'un biais systématique dû à l'extension vers les basses énergies finales de la bande $\mathrm{du}$ spectre voisine du point expérimental à corriger. En effet, l'approximation consistant à assimiler une bande d'énergie du spectre à un pic centré sur le milieu de la bande est dans ce cas d'autant plus mauvaise que $\Delta E$ est grand.

Nous retrouvons le même phénomène quand nous étudions l'influence du pas d'itération $\Delta$ sur la correction d'un spectre correspondant à une valeur de $\Delta E$ déterminée (Fig. 10). Le spectre corrigé est reproductible tant que la valeur de $\Delta$ n'est pas trop différente de la valeur de la résolution expérimentale $\Delta E$.

IV.2. ETUDE DE LA PORTION DU SPECTRE CORRESPONDANT AUX GRANDES ÉNERGIES D'EXCITATION. Nous avons étudié sur un spectre d'électrons diffusés à un angle de $60^{\circ}$, pour une énergie incidente $E_{\mathrm{i}}=213,4 \mathrm{MeV}$, la portion du spectre correspondant aux grandes énergies d'excitation $(\omega>120 \mathrm{MeV})$. Dans cette zone d'énergie, la section efficace expéri- 


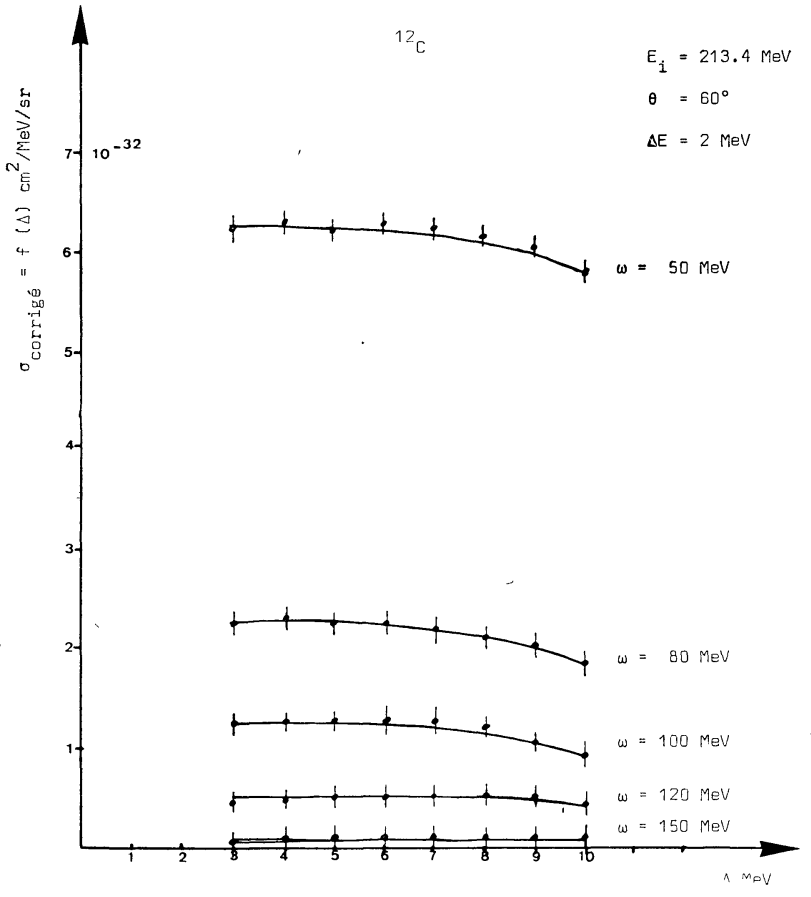

FIG. 10. - Section efficace corrigée pour divers $\omega$ en fonction de $\Delta$, pas d'itération du programme de déconvolution.

mentale est due essentiellement à l'extension du pic élastique, à l'exclusion de tout autre phénomène, tant que l'électroproduction peut être considérée comme négligeable. En effet, les conditions cinématiques ont été choisies telles que la diffusion quasi élastique et son extension vers les basses énergies finales soient très faibles à partir de $120 \mathrm{MeV}$.

Nous avons ainsi accès d'une manière directe à la somme des contributions des diagrammes $(a)$ et $(b)$ de la figure 3 , ce qui va nous permettre de vérifier la validité des formules employées. Si toutefois, pour d'autres conditions cinématiques la contribution de l'électroproduction des pions s'avère non négligeable, il est possible d'en évaluer la contribution grâce aux expressions proposées par Czyz et Walecka [20] pour l'électroproduction près du seuil, ou par Moniz [21] pour l'électroproduction à la résonance 3-3.

La section efficace étant dominée par la contribution radiative dans cette région nous pouvons donc écrire :

$$
\sigma_{\mathrm{ex}}=Q_{1}+Q_{2} \cdot t
$$

où $Q_{1}$ correspond à la contribution du rayonnement de freinage interne alors que $Q_{2}$ correspond au ayonnement de freinage externe et à l'ionisation dans le matériau cible, $t$ étant l'épaisseur de la cible.

Ces deux termes $Q_{1}$ et $Q_{2}$ ont été séparés expérimentalement en utilisant plusieurs épaisseurs de cible. Les courbes $(a)$ et $(b)$ de la figure 11 montrent qu'il existe un bon accord entre la valeur expérimentale de $Q_{1}$ et celle calculée au moyen des expression (10), (1) et (12). Remarquons toutefois que la perte d'énergie par rayonnement étant du même ordre de gran- deur que l'énergie des électrons incidents $\left(k / E_{1}>0,6\right)$, l'approximation du « pic » (courbe $(a)$ ) conduit à une valeur beaucoup trop grande de $Q_{1}$. Nous vérifions aussi une conclusion qui avait été déjà signalée par de nombreux auteurs [5], [14], [31]. Sur la figure 12, nous avons porté la valeur expérimentale du terme $Q_{2}$, ainsi que les résultats du calcul effectué au moyen des expressions (13) et (14). Un excellent accord est obtenu, aussi bien ce en qui concerne la valeur absolue du terme $Q_{2}$, que de sa variation en fonction de $\omega$.

Nous avons d'autre part vérifié que l'ensemble des corrections effectuées est correct en étudiant dans

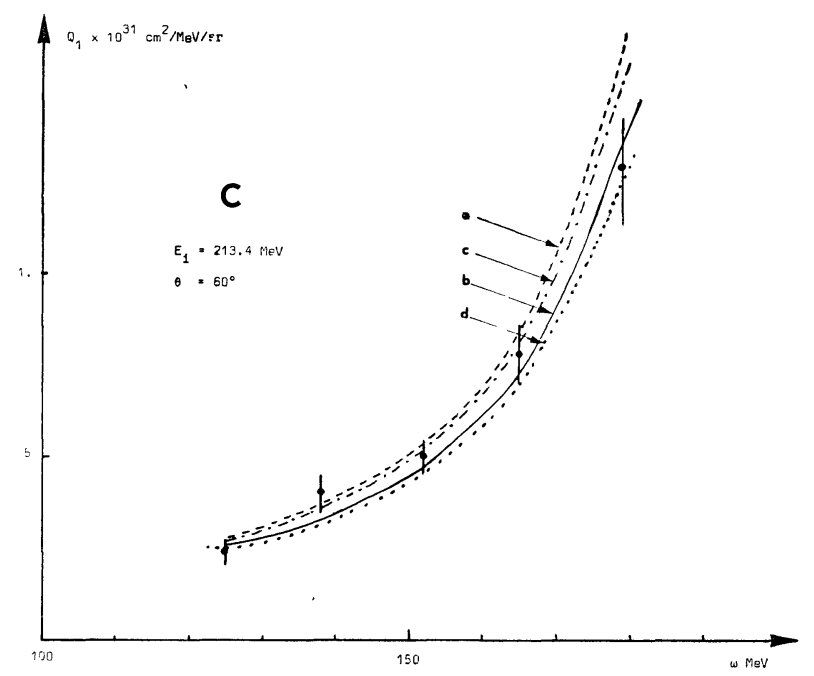

Fig. 11. - Résultats expérimentaux et calcul du terme $Q_{1}$ (défini éq. (20)) pour le spectre de diffusion sur le carbone correspondant à $E_{i}=213,4 \mathrm{MeV}$ et $\theta=60^{\circ}$.

(a) $--\ldots-11^{\text {re }}$ Born approximation du pic (éq. (25))

(b) $1^{\text {re }}$ Born sans approximation (éq. (26))

(c) $-.+\cdots \cdot-\ldots \cdot 2^{\mathrm{e}}$ Born approximation du pic (éq. (27))

(d) $\ldots \ldots \ldots \ldots 2^{\mathrm{e}}$ Born sans approximation du pic (éq. (28)).

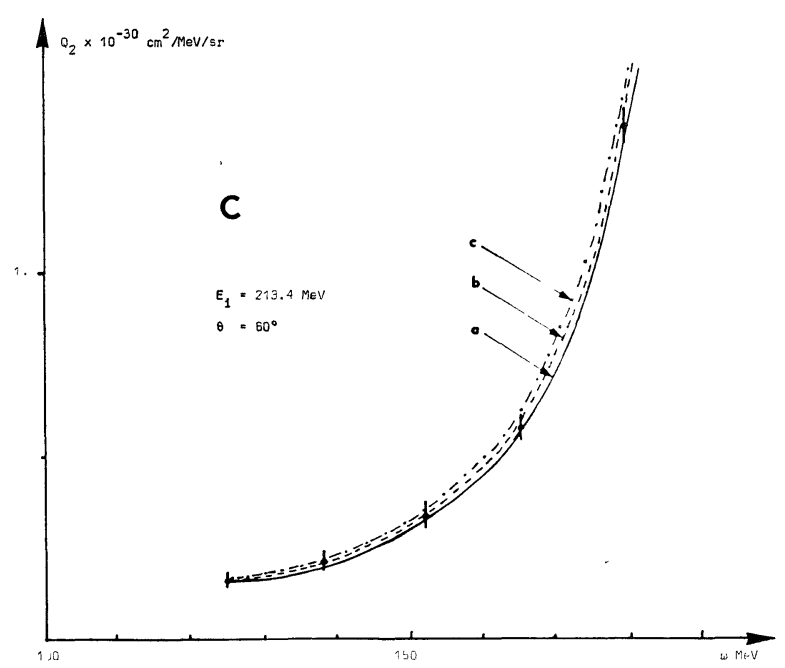

FIG. 12. - Idem pour le terme $Q_{2}$.

(a) $1^{\text {re }}$ Born (éq. (13)).

(b) $-\ldots-\ldots 2^{\mathrm{e}}$ Born Olsen (réf. [28]).

(c) - - . - . - - 2 $2^{\mathrm{e}}$ Born Roche (réf. [29]). 
cette zone d'énergie d'excitation la grandeur de la section efficace corrigée qui doit être statistiquement nulle.

Nous avons porté sur la figure 13, pour un spectre de diffusion correspondant à $E_{\mathbf{i}}=213,4 \mathrm{MeV}$ et $\theta=60^{\circ}$, la distribution en amplitude de la section efficace expérimentale corrigée pour tous les points expérimentaux tels que $120<\omega<180 \mathrm{MeV}$. La

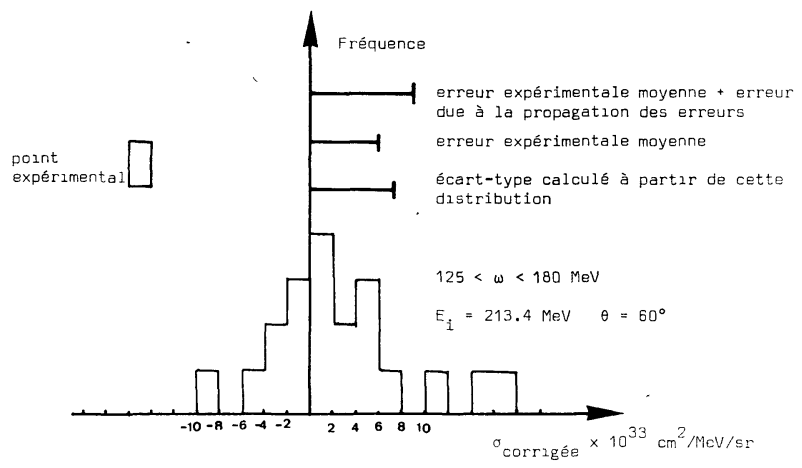

Fig. 13. - Histogramme de la section efficace corrigée pour

$$
125<\omega<180 \mathrm{MeV} \text {. }
$$

forme, la moyenne et l'écart-type de cette distribution montrent que celle-ci est compatible avec une distribution gaussienne centrée sur une valeur de la section efficace égale à zéro. De plus, la valeur de l'écarttype de cette distribution est en accord avec celle calculée en supposant une propagation quadratique de l'erreur expérimentale (éq. 18). Il faut cependant que la queue "inélastique » est peu importante dans cette région d'énergie d'excitation, le terme $\left(\Sigma\left(\Delta T_{m j}\right)\right)^{2}$ de l'éq. (18) étant très faible par rapport au terme $\left(\Delta \sigma_{\mathrm{ex}}\right)^{2}$. Par contre, dans le cas où la contribution inélastique à la queue radiative est importante, un calcul de simulation nous a permis de vérifier que l'erreur calculée au moyen de l'expression (18) conduit à une valeur trop grande de l'erreur expérimentale totale (Fig. 14).

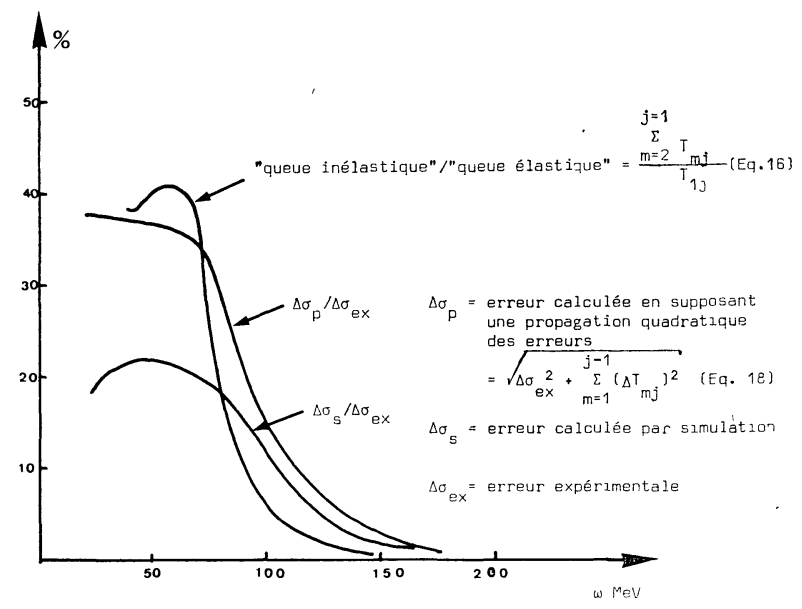

FIG. 14. - Variations comparées de l'importance relative de la «queue inélastique», et de l'erreur calculée d'une part par propagation de l'erreur quadratique et d'autre part, par simulation.
V. Vérifications expérimentales des corrections apportées à la première approximation de Born. - Toutes les formules utilisées pour le calcul des queues radiatives sont établies à partir de la section efficace dite de Bethe-Heitler [15] pour le rayonnement de freinage. Or, celle-ci a été calculée en ne prenant en compte que les termes correspondant à l'échange d'un seul photon virtuel (première approximation de Born). L'expérience (16) a montré qu'une telle approximation n'est plus valable pour les noyaux lourds $(Z \alpha>1)$.

Un certain nombre d'auteurs' [25], [27] ont établi des expressions de la section efficace de rayonnement de freinage d'électrons diffusés dans le champ coulombien d'un noyau ponctuel en tenant compte des termes avec échange de deux photons (deuxième approximation de Born). Mais ce n'est que récemment que l'intégration de cette section efficace sur les angles d'émission du photon a été réalisée par Borie [19]. Elle obtient ainsi une expression particulièrement simple de la correction à apporter à la diffusion nucléaire suivie ou précédée de l'émission d'un photon réel. Toutefois, elle s'est limitée au calcul du terme $P$ de l'expression (10) (approximation de pic) car celui du terme $B$ implique le calcul d'intégrales beaucoup trop compliquées.

Posons :

$$
\sigma=\sigma_{1 B}+\sigma_{2 B}
$$

avec :

$\sigma_{1 B}=$ section efficace de diffusion nucléaire suivie ou précédée de l'émission d'un photon, calculée en première approximation de Born.

$\sigma_{2 B}=$ terme correctif tenant compte de l'échange de deux photons.

Borie démontre que dans le cas de la diffusion élastique accompagnée de l'émission d'un photon réel, il existe une relation simple entre $\sigma_{1 B}$ et $\sigma_{2 B}$. Soit :

$$
\frac{\sigma_{2 B}}{\sigma_{1 B}}=\pi \alpha Z \frac{\sin \theta / 2}{1+\sin \theta / 2} .
$$

Le résultat est donné sous cette forme car on peut considérer que ce rapport est sans doute moins sensible que la section efficace elle-même à la distribution finie de charge du noyau. Toutefois, il est possible que ce rapport ne soit pas indépendant de l'énergie dans le cas d'un noyau d'extension finie. Notons toutefois que ce résultat obtenu dans le cas de la diffusion coulombienne accompagnée par l'émission d'un photon réel est identique à celui obtenu par Dalitz [32] dans le cas de la diffusion coulombienne simple.

Pour tenir compte des corrections coulombiennes dans le calcul du rayonnement de freinage externe nous avons employé l'expression donnée par Olsen [28]. Cette expression intégrée analytiquement sur les angles d'émission du photon tient compte de l'effet d'écran des électrons atomiques et emploie l'approximation ultra-relativiste $\left(E_{\mathrm{i}}, E_{\mathrm{f}}, k \gg m c^{2}\right)$. 
Nous avons aussi utilisé la formule donnée par Roche et al. [29]. Ces auteurs incluent dans leur calcul, par rapport à celui d'Olsen, des éléments de matrice d'ordre supérieur provenant de la décomposition des fonctions d'ondes en harmoniques sphériques. Toutefois, l'intégration analytique de cette expression sur les angles d'émission du photon n'étant pas réalisable, nous l'avons effectuée numériquement. L'effet d'écran non inclus dans le calcul, a été introduit en appliquant au résultat le même facteur correctif que pour les expressions sans corrections coulombiennes. Nous pouvons appliquer cette méthode, car pour les énergies considérées, l'effet d'écran est faible $(<15 \%)$ et nous pouvons alors admettre que les corrections coulombiennes et d'effet d'écran sont additives.

Sur la figure 15, sont comparés les résultats des

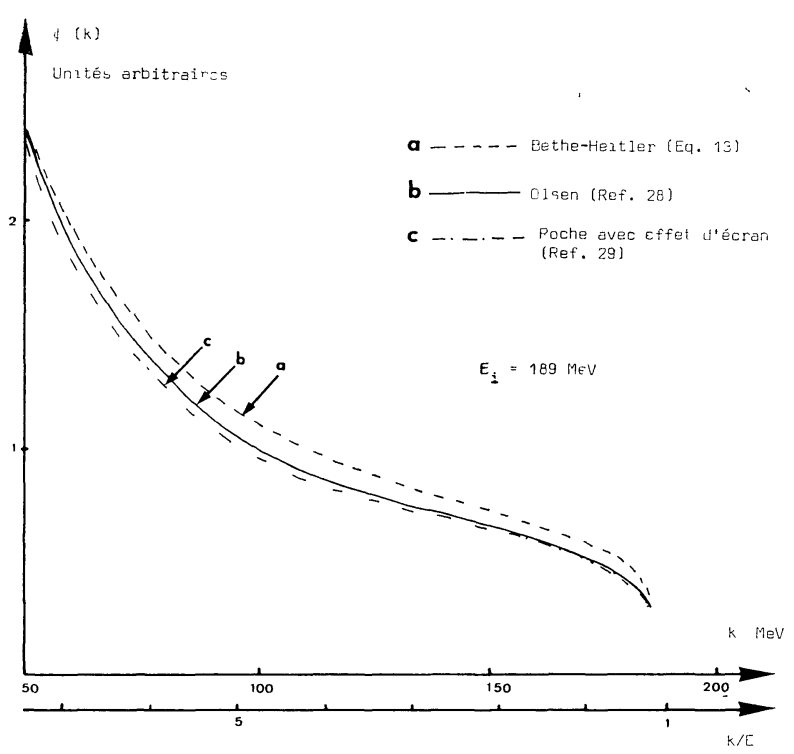

FIg. 15. - Divers calculs de la probabilité $\varphi(k) \mathrm{d} k=f(k) \mathrm{d} k$ qu'un électron d'énergie $189 \mathrm{MeV}$ émette par rayonnement de freinage sur du bismuth $(Z=83)$ un photon d'énergie comprise entre $k$ et $k+\mathrm{d} k$.

différentes expériences pour le calcul de la probabilité $\varphi(k)$. d $k$ d'émission d'un photon d'énergie comprise entre $k$ et $k+\mathrm{d} k$ par rayonnement de freinage d'un électron de $189 \mathrm{MeV}$ sur le bismuth 209.

On voit que les corrections apportées sont faibles: la plus grande divergence observée entre ces divers résultats est de l'ordre de $12 \%$. Ceci justifie notamment a posteriori l'hypothèse émise ci-dessus au sujet du caractère additif des corrections coulombiennes et d'écran.

La contribution à la queue radiative due à la perte d'énergie des électrons par ionisation sera calculée comme indiqué dans la partie II (éq. 8). Remarquons ici que d'une part ce terme varie comme $Z / A$, alors que le terme de rayonnement de freinage varie comme $Z^{2} / A$ et que d'autre part, aux énergies considérées, sa contribution est toujours faible devant celle du rayonnement de freinage $(<2 \%)$.

Afin de vérifier si l'ensemble de cette approche est correct, nous avons réalisé des mesures sur le bismuth 209 et l'étain 80 en plus des mesures sur le carbone 12 précédemment citées. Afin que la diffusion inélastique et son extension vers les basses énergies finales ne masque pas partiellement le phénomène radiatif, nous avons étudié seulement la portion des spectres de diffusion correspondant aux grandes énergies d'excitation.

Toutefois, cette méthode présente l'inconvénient de nous placer dans des conditions telles que l'approximation du pic est mauvaise. Or, appliquant la correction du second ordre, définie éq. (22), uniquement sur le terme $P$ de l'éq. (10), nous introduisons un biais dû au fait que le terme $B$ est calculé en première approximation de Born.

Ecrivant l'éq. (10) sous la forme:

$$
\sigma_{1 B}=K(P+B) \quad \sigma_{1 B}^{\text {schiff }}=K \cdot P
$$

avec :

$$
K=\frac{1}{2 \pi} \cdot \frac{e^{2}}{\hbar c} \cdot\left(\frac{Z e^{2}}{m c^{2}}\right)^{2} \frac{1}{k} \cdot \frac{1}{E_{\mathrm{i}}^{2}}
$$

le facteur correctif de l'expression (21) deviendra:

$$
\frac{\sigma_{2 B}}{\sigma_{1 B}^{\text {schiff }}}=\lambda=\pi \alpha Z \frac{\sin \theta / 2}{1+\sin \theta / 2} .
$$

La section efficace de rayonnement de freinage interne devient donc :

$$
\sigma=\sigma_{1 B}+\sigma_{2 B}=K(P+B)+\lambda K P .
$$

Toutes les expériences sur le ${ }^{209} \mathrm{Bi}$ ont été effectuées en laissant l'angle de diffusion $\theta$ constant et égal à $76,5^{\circ}$, tandis que celles sur le carbone et l'étain correspondent à un angle $\theta=60^{\circ}$.

Nous avons comme au paragraphe IV.2, par des mesures avec des épaisseurs de cibles différentes, séparé expérimentalement les contributions $Q_{1}$ et $Q_{2}$ correspondant respectivement au rayonnement de freinage interne et externe :

$$
\sigma=Q_{1}+Q_{2} . t
$$

Sur les figures $11,16,17,18$, les valeurs expérimentales de la quantité $Q_{1}$ sont comparées aux résultats des différents calculs décrits ci-dessus.

Pour chacun de ces cas, nous avons calculé :

a) $Q_{1}$ en première approximation de Born et avec approximation du pic. Soit :

$$
Q_{1}=K P \text {. }
$$

b) $Q_{1}$ en première approximation de Born sans approximation de pic. Soit :

$$
Q_{1}=K(P+B) .
$$

c) $Q_{1}$ en deuxième approximation de Born, le terme correctif $\lambda$ étant appliqué sur la somme $(P+B)$. Soit :

$$
Q_{1}=K \lambda(P+B) \text {. }
$$


d) $Q_{1}$ en deuxième approximation de Born, le terme correctif $\lambda$ étant appliqué sur le terme $P$ seulement. Soit :

$$
Q_{1}=K(P+B)+\lambda K P .
$$

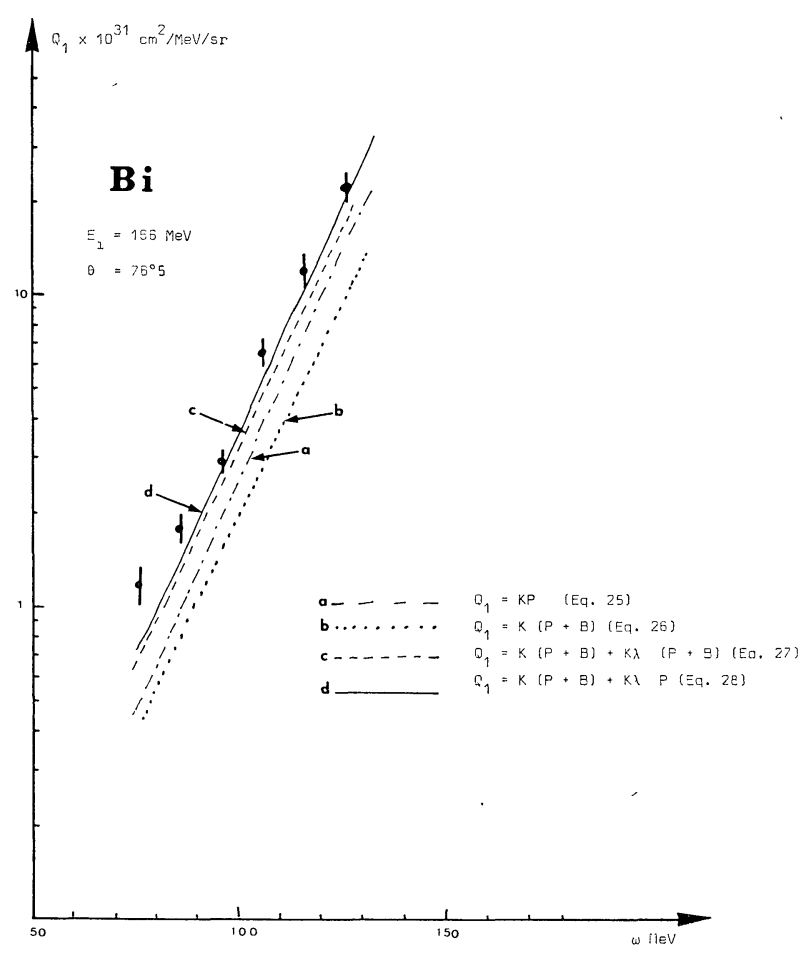

FIG. 16. - Résultats expérimentaux et calculs du térme $Q_{1}$ pour le spectre de diffusion sur le bismuth correspondant à $E_{i}=166 \mathrm{MeV}$ et $\theta=76,5^{\circ}$. Voir dans le texte la signification des différentes courbes $(\S \mathrm{V})$.

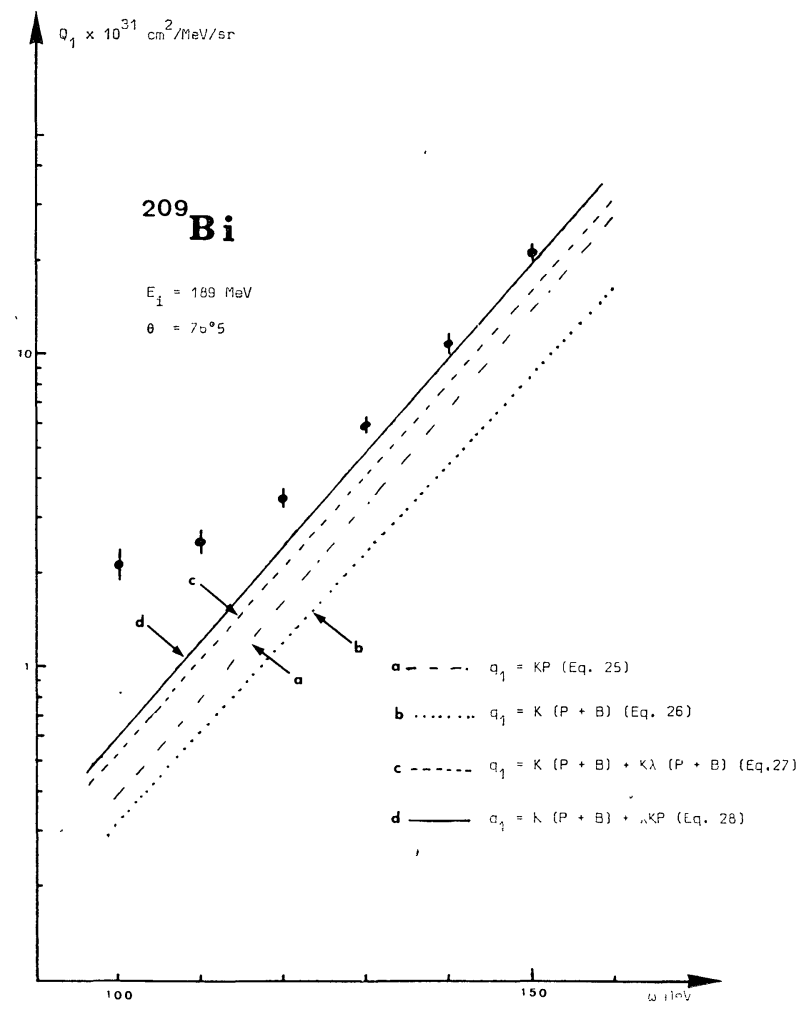

FIG. 17. - Idem pour $E_{1}=189 \mathrm{MeV}$ et $\theta=76,5^{\circ}$.

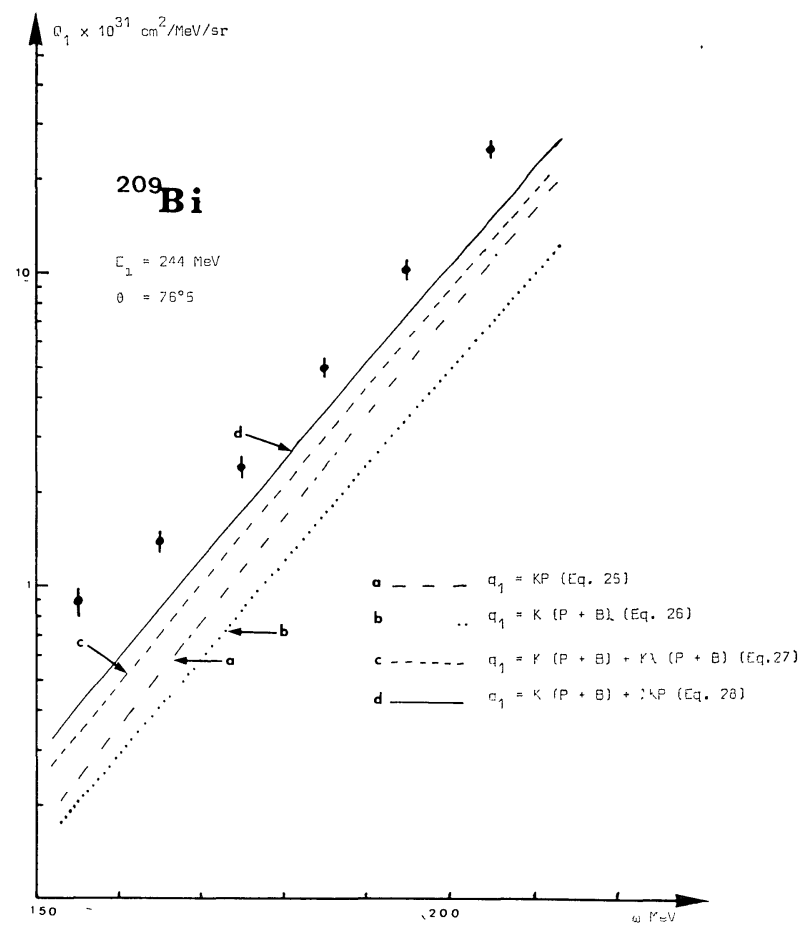

FIG. 18. - Idem pour $E_{1}=244 \mathrm{MeV}$ et $\theta=76,5^{\circ}$.

Rappelons que seule la partie des spectres correspondant à une énergie d'excitation suffisamment grande est à comparer aux calculs de queues radiatives. En effet, pour les énergies d'excitation plus faibles, il y a une contribution de la diffusion quasi élastique à la section efficace mesurée, contribution que nous ne pouvons séparer expérimentalement du phénomène radiatif.

L'ensemble de ces calculs, a été conduit en utilisant pour les facteurs de forme élastique, les expressions données par Petkov [30]. Notons, toutefois, que le choix de la forme analytique du facteur de forme élastique n'est pas critique, car aux transferts de quantité de mouvement considérés $\left(q \ll 1,5 \mathrm{fm}^{-1}\right)$ toutes les expressions proposées dans la littérature donnent le même résultat.

L'analyse de ces résultats montre que, si l'utilisation de la première approximation de Born conduit à un accord satisfaisant en ce qui concerne le carbone, à condition toutefois de ne pas utiliser l'approximation du pic, elle introduit un biais systématique de plus de $50 \%$ pour un élément lourd tel que le bismuth. L'introduction du facteur correctif $\lambda$ défini par l'éq. (23) amène un accord à peu près satisfaisant entre expérience et calcul. Il semble toutefois que la validité de ce terme correctif soit fonction de l'énergie. En effet, l'étude de la variation du rapport entre calcul en deuxième approximation de Born et expérience (Fig. 19) montre que celui-ci augmente avec l'énergie incidente.

Ceci est vraisemblablement dû au fait que le calcul du facteur correctif $\lambda$ est réalisé en choisissant une distribution de charge ponctuelle pour le noyau. De plus, on peut s'attendre à un biais systématique diffi- 


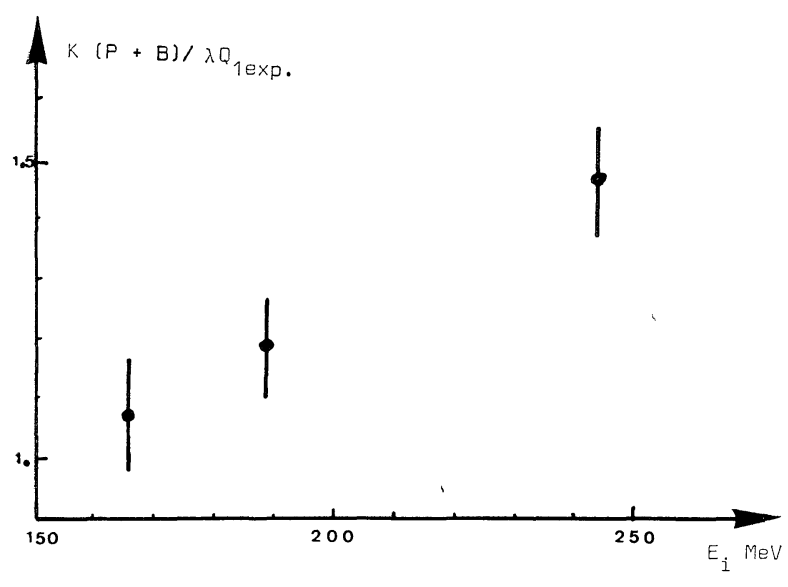

FIg. 19. - Variation en fonction de l'énergie incidente, de la divergence observée entre les résultats expérimentaux et le calcul effectué en deuxième approximation de Born.

cilement évaluable dû à l'absence d'un facteur correctif du second ordre pour le terme $B$ de l'éq. (10).

Les figures $12,20,21$ et 22 donnent les variations du terme $Q_{2}$ obtenues respectivement pour les noyaux de carbone et de bismuth.

Pour chacun de ces cas, nous avons calculé :

a) $Q_{2}$ par la formule de Bethe-Heitler sans correction coulombienne et avec effet d'écran;

b) $Q_{2}$ par la formule d'Olsen avec correction coulombienne et effet d'écran;

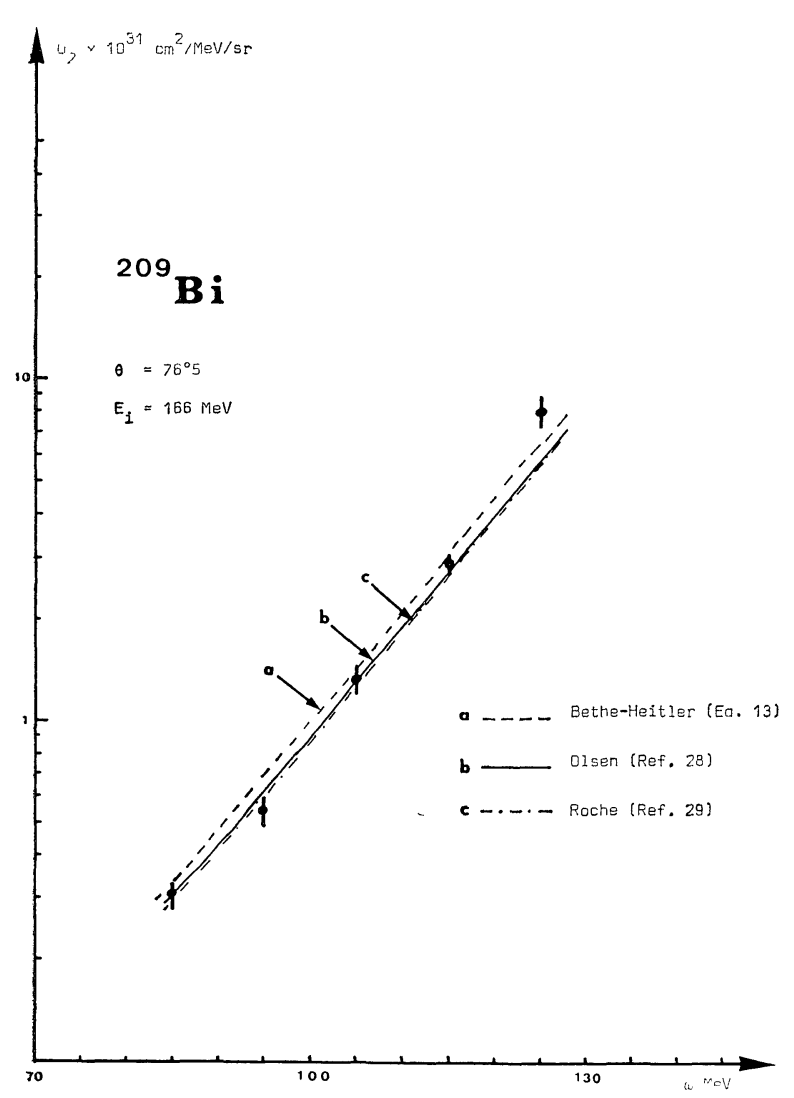

FIG. 20. - Résultats expérimentaux et calculs du terme $Q_{2}$ pour le spectre de diffusion sur le bismuth correspondant à $E_{i}=166 \mathrm{MeV}$ et $\theta=76,5^{\circ}$.

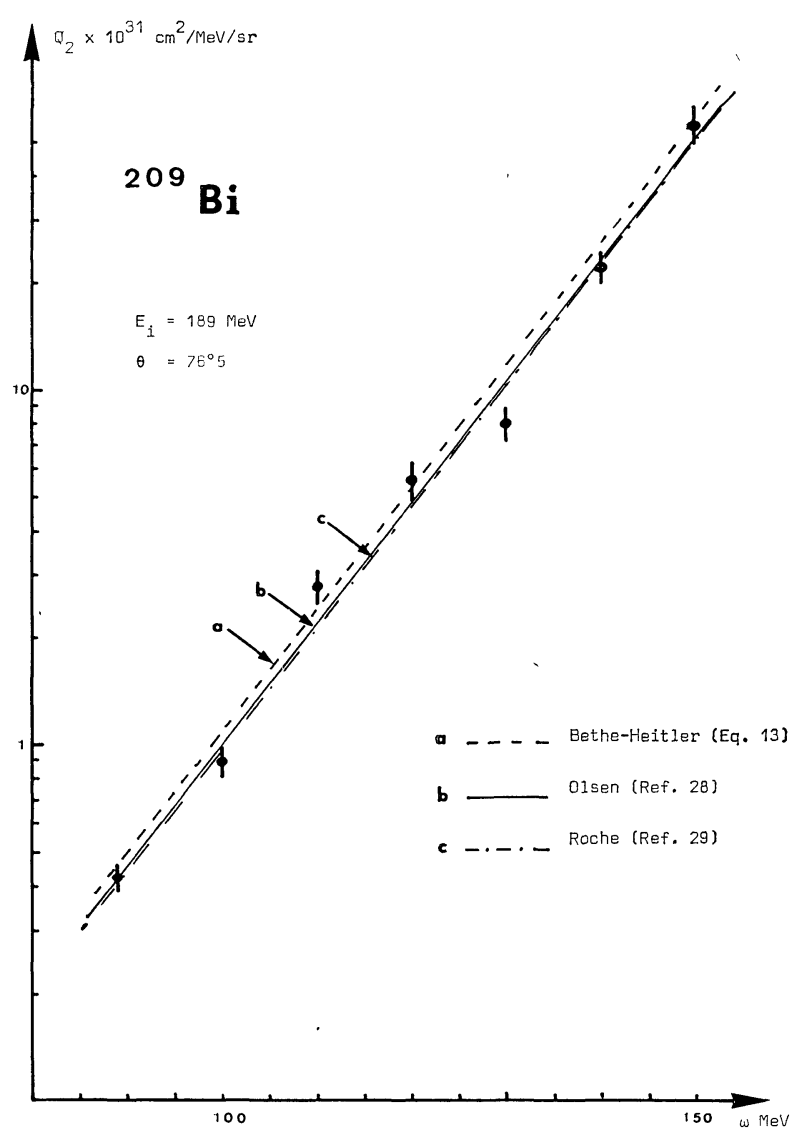

FIG. 21. - Idem pour $E_{i}=189 \mathrm{MeV}$ et $\theta=76,5^{\circ}$.

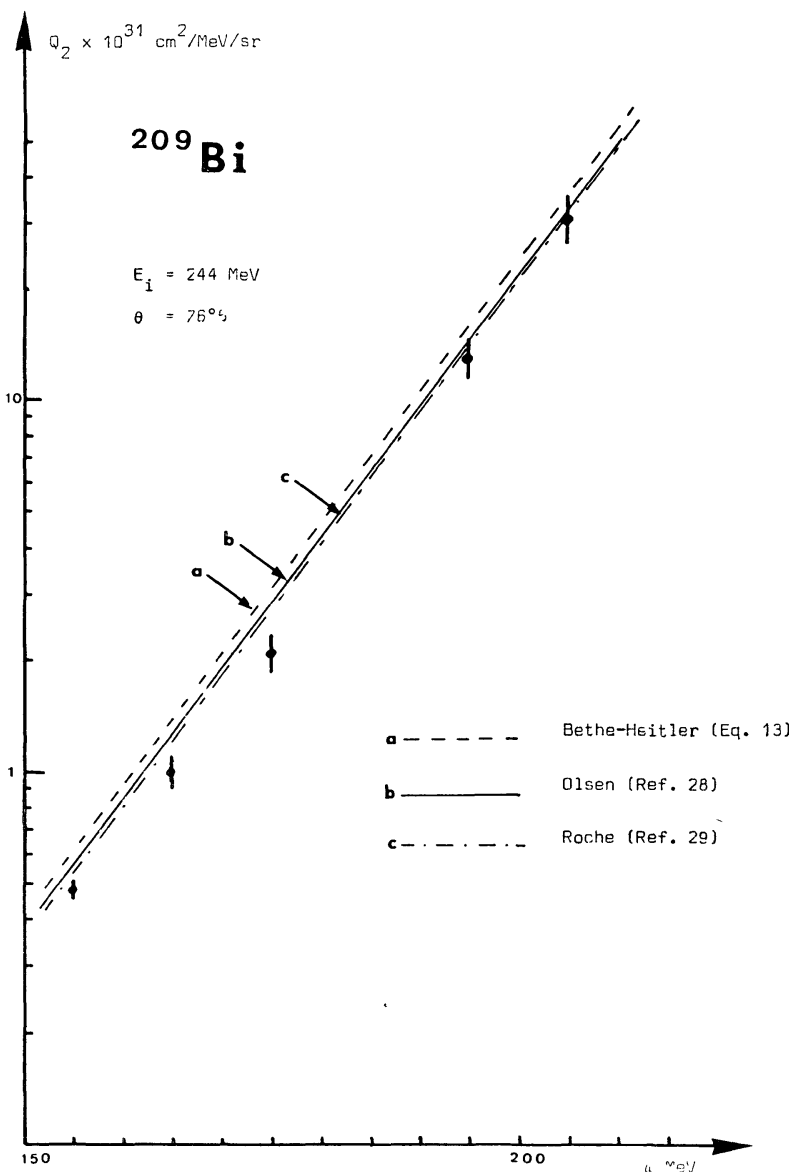

FIG. 22. - Idem pour $E_{i}=244 \mathrm{MeV}$ et $\theta=76,5^{\circ}$. 
c) $Q_{2}$ par la formule de Roche intégrée numériquement avec introduction de l'effet d'écran.

L'analyse des résultats montre que, même dans le cas d'un noyau lourd tel que le bismuth, le calcul en première approximation de Born conduit à des résultats satisfaisants, la divergence entre expérience et calcul n'excédant pas $10 \%$. Cet accord est dû au fait que l'angle d'émission du photon produit par rayonnement de freinage externe est toujours faible dès l'instant où l'énergie de l'électron est grande $\left(\theta_{\text {photon }}<1 / E\right)$. Toutefois, les calculs n'utilisant pas la première approximation de Born apportent à celle-ci une correction supérieure à $5 \%$ dans le cas du bismuth, l'accord entre calcul et expérience étant alors réalisé dans la limite des erreurs expérimentales.

Nous avons de plus mesuré pour une énergie incidente de $213,4 \mathrm{MeV}$ et $\theta=60^{\circ}$, un spectre de diffusion sur l'étain, noyau intermédiaire entre le carbone et le bismuth. Sur la figure 23, nous comparons les résultats expérimentaux aux calculs effectués respectivement au premier et au second ordre. Les calculs du second ordre correspondent à l'emploi pour le rayonnement de freinage interne du facteur $\lambda$ de l'éq. (23) appliqué uniquement sur le terme $P$. Pour le rayonnement de freinage externe, nous avons employé ici la formule d'Olsen.

Les calculs du second ordre conduisent à un accord

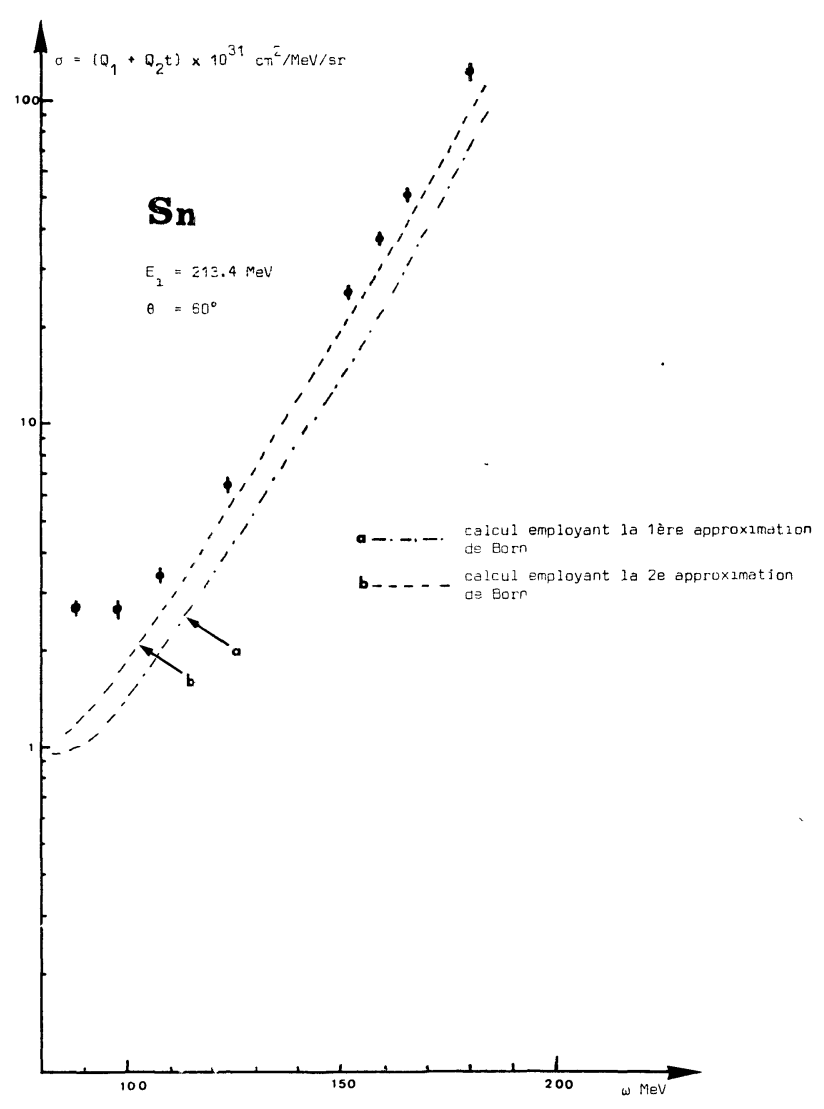

FIG. 23. - Résultats expérimentaux et calculs de la section efficace pour le spectre de diffusion sur l'étain correspondant à

$$
E_{i}=213,4 \mathrm{MeV} \text { et } \theta=60^{\circ} \text {. }
$$

satisfaisant en ce qui concerne la forme de la remontée ne la queue élastique et à une légère divergence de 5 à $10 \%$ en ce qui concerne la valeur absolue de la section efficace radiative.

Conclusion. - La méthode de "déconvolution" que nous avons utilisée donne de bons résultats pour l'analyse des spectres de diffusion des électrons sur les noyaux légers tels que le carbone. En effet, les diverses vérifications expérimentales que nous avons réalisées ne montrent aucune distorsion décelable dans les spectres corrigés. Les expressions théoriques de la section efficace radiative établies en première approximation de Born donnent des résultats en bon accord avec les expériences dans les limites des erreurs statistiques à savoir quelques pour-cent.

Cependant, un certain nombre de précautions doivent être prises aussi bien lors de la collection des données que pour leur analyse :

- l'épaisseur de cible doit être inférieure à un centième de longueur de radiation afin d'une part, d'éviter une trop grande dispersion des trajets des électrons, d'autre part, d'être sûr que la contribution due à l'émission de deux photons soit négligeable, - le choix des spectres constituant le triangle canonique doit être fait très judicieusement pour limiter les imprécisions dues aux interpolations; ce choix dépend en particulier de l'énergie d'excitation des points expérimentaux à corriger,

- le pas d'itération $\Delta$ du programme d'analyse doit être choisi voisin de la résolution expérimentale $\Delta E$,

- l'approximation du pic ne peut pas être utilisée pour des pertes d'énergie élevées $(k / E<0,3)$ dans le calcul de l'extension des pics élastiques; son emploi se justifie pour les pics inélastiques quand la variation de la section efficace de l'énergie d'excitation est lente.

Par contre, l'analyse des spectres de diffusion sur un noyau lourd montre que l'emploi des expressions établies en première approximation de Born conduit à des résultats en désaccord avec les résultats expérimentaux. Cette divergence, surtout très importante en ce qui concerne la contribution du rayonnement de freinage interne, peut atteindre $50 \%$. Nous avons montré que l'utilisation des expressions établies en deuxième approximation de Born par Borie pour le pic élastique améliore sensiblement le résultat. Ces résultats démontrent que l'expression obtenue dans le cadre de la première approximation n'est valable que pour des noyaux cibles de numéro atomique inférieur à 20 et des angles de diffusion compris entre $50^{\circ}$ et $150^{\circ}$. Toutefois, l'expression proposée par Borie a été établie dans le cas de l'approximation du pic et n'est donc utilisable que pour de faibles pertes d'énergie.

Par contre, en ce qui concerne la contribution due au rayonnement de freinage externe, les résultats obtenus montrent que l'utilisation de formules établies en première approximation de Born donnent 
un accord satisfaisant $(\sim 10 \%)$ avec les résultats expérimentaux, les termes du second ordre apportent une correction de l'ordre de $5 \%$ pour un noyau très lourd comme le bismuth.

La contribution du rayonnement de freinage interne étant cependant toujours très grande, l'analyse précise des spectres de diffusion inélastique et quasi élastique sur les noyaux lourds est rendue difficile par l'absence, dans la littérature, d'expressions établies en deuxième approximation de Born.

Remerciements. - Les auteurs souhaitent remercier le Dr Borie et le Dr Moniz pour les fructueuses discussions qu'ils ont eues et remercient le personnel de l'accélérateur linéaire d'Orsay d'avoir permis la réalisation de cette série d'expériences.

\section{Bibliographie}

[1] Walecka (J. D.) et De Forest (T.), Adv. in Phys., 1966, 57, 1 .

Chemtob (M.), Note CEA, 1966, 623.

[2] Kendall (H. W.) et Isabelle (D.), Bull. Am. Phys. Soc., 1964, 9, 94.

[3] Crannell (H.), Nucl. Inst. and Meth., 1969, 71, 208.

[4] Ganichot (D.), Grossetete (B.) et Isabelle (D. B.), Nucl. Inst. and Meth., 1971, 96, 525-537.

[5] Mo (L. W.) et Tsaï (Y. S.), Rev. Mod. Phys., 1969, 41, 205.

[6] Schwinger (J.), Phys. Rev., 1949, 15, 898.

[7] Yennie (D. R.) et Suura (H.), Phys. Rev., 1957, 105,1378 .

Yennie (D. R.), Frautschi (S. C.) et SuUra (H.), Ann. of Phys., New York, 1961, 13, 379.

[8] Lacoste (F.), Thèse de Doctorat d'Etat, Université de Paris, 1962.

[9] Meister (N. J.) et Griffy (J. A.), Phys. Rev., 1964, B 133, 1032.

[10] Maximon (L. C.), Rev. Mod. Phys., 1969, 41, 193.

[11] Rossi (B.), High Energy Particles, Prentice Hall, Inc. Englewood Cliffs, N. J., 1952.

[12] Landau (L.), J. Phys., USSR, 1944, 8, 201.

[13] Nguyen Ngoc (N.), Ann. de Phys., 1965, 10, 315.

[14] Maximon (L.) et Isabelle (D. B.), Phys. Rev., 1964, B 136, 674.
[15] Bethe (H.) et Heitler (W.), Proc. R. Soc., 1934, A 146, 83.

[16] Koch (H. W.) et Motz (J. W.), Rev. Mod. Phys., 1959, 31, 920.

[17] Moller (C.), Ann. Phys., 1932, 14, 531.

[18] Schiff (L. I.), Phys. Rev., 1952, 87, 750.

[19] Borie (E.), Lett. Nuovo Cim., 1971, 1, 106.

[20] Czyz (W.) et Walecka (J. D.), Nucl. Phys., 1964, $51,312$.

[21] Moniz (E. J.), Phys. Rev., 1969, 184, 1154.

[22] Perez y Jorba (J.), J. Physique Radium, 1961, 22, 323.

[23] Segre (E.), Experimental Nuclear Physics, John Wiley and Sons, Inc., New York, 1953.

[24] Uberall (H.), NRL Report, 1970, 7080.

[25] Kacser (C.), Proc. Roy. Soc., 1959, A 253, 103.

[26] Derk (R. T.), Moroi (D. S.) et Alling (W. R.), Nucl. Phys., 1969, A 133, 321.

[27] BORIE (E.), NBS Report, 1970, 10321 (non publié).

[28] Olsen (H.), Phys. Rev., 1955, 99, 1335.

[29] Roche (G.), Ducos (C.) et Proriol (J.), Soumis à Phys. Rev.

[30] Petkov (I. Z.), Sov. Journal of Nucl. Phys., 1967, 4, 411.

[31] Alles Borelli (A.), Phys. Lett., 1971, 36B, 149. 